\title{
Hierarchical assemblies of molecular frameworks-MOF-on-MOF epitaxial heterostructures
}

\author{
Ritesh Haldar and Christof Wöll $(\square)$ \\ Institute of Functional Interfaces (IFG), Karlsruhe Institute of Technology (KIT), Hermann-von-Helmholtz-Platz, 176344 Eggenstein- \\ Leopoldshafen, Germany
}

(C) The Author(s) 2020

Received: 4 March 2020 / Revised: 31 May 2020 / Accepted: 26 June 2020

\begin{abstract}
Functional, porous metal-organic frameworks (MOFs) have attracted much attention as a very flexible class of crystalline, porous materials. For more advanced applications that exploit photophysical properties, the fabrication of hierarchical assemblies, including the creation of MOF/MOF heterointerfaces, is important. For the manufacturing of superstructures with length scales well beyond that of the MOF pore size, layer-by-layer (Ibl) methods are particularly attractive. These allow the isoreticular approach to be extended to superstructures with micrometer length scales, a range that is not accessible using conventional MOF design. The Ibl approach further substantially extends the compositional diversity in MOFs. At the same time, the favorable elastic properties of MOFs allow for heteroepitaxial growth, even in the case of lattice misfits as large as $20 \%$. While the MOF-on-MOF approach to designing multicomponent superstructures with synergistic multifunctionality can also be realized with sophisticated solvothermal synthesis schemes, the $\mathrm{lbl}$ (or liquid-phase epitaxy) approach carries substantial advantages, in particular when it comes to the integration of such MOF superstructures into optical or electronic devices. While the structure vertical to the substrate can be adjusted using the $\mathrm{lbl}$ method, photolithographic methods can be used for lateral structuring. In this review, we will discuss the lbl liquid-phase epitaxy approach to growing surface-anchored MOF thins films (SURMOFs) as well as other relevant one-pot synthesis methods for constructing such hierarchically designed structures and their emerging applications.
\end{abstract}

\section{KEYWORDS}

metal-organic framework (MOF) heterostructure, hierarchical assembly, epitaxy, layer-by-layer growth, surface-anchored MOFs

\section{Introduction}

Over the past two decades in the field of coordination chemistry, the art of linking multitopic molecular building blocks to create crystalline materials with designable void space (porosity) has been developed to very high standards, providing a huge chemical space. Net design or reticular chemistry $[1,2]$, principally based on the simple combining of metal (node)organic (linker) constituents, have yielded > 100,000 new crystalline structures with an enormous variety of different structural topologies and physical/chemical properties [3-5]. A very straightforward reticular design approach can provide exquisite control over the net topology and functionality of these materials. These intrinsically porous crystalline coordination networks, also known as metal-organic frameworks (MOFs) or porous coordination polymers (PCPs), have unsurpassed (by other materials) surface areas $\left(>7,000 \mathrm{~m}^{2} / \mathrm{g}\right)$ [6]. They have primarily found applications in the fields of gas storage $[7,8]$, separation $[9,10]$, and catalysis $[11,12]$. However, the photophysical and electronic properties of these materials are also exciting and have triggered interest in the areas of optoelectronics [13-15], sensing [16], and biosciences [17, 18].

The isoreticular synthesis approach [19] has been a powerful tool for designing and expanding the porosity and functionality of MOFs. Using this strategy, without altering the structural topology, the periodicity and thus the pore size of a framework structure can be expanded by adjusting the length of the organic linkers to appropriate values. Attaching side groups to the organic linkers allows the inner walls of the pores to be decorated with many different types of chemical functionalities, thus yielding huge functional diversity [20]. While pore sizes as large as $\sim 10 \mathrm{~nm}$ have been realized using long ditopic linkers (for IRMOF-74) [21], the realization of superstructures in the micrometer regime, required for optical applications for example, is not straightforward. Clearly, for the manufacturing of MOF assemblies consisting of spatially separated regions with different functionalities or pore sizes, approaches beyond solvothermal reaction procedures are required. The fabrication of structurally well-defined $\mathrm{MOF} / \mathrm{MOF}$ heterointerfaces, e.g., required for optical (upconversion) and photovoltaic (charge separation) applications, also represents challenges which cannot be overcome with conventional synthesis methods.

The fabrication of such MOF heterostructures becomes straightforward if MOFs are grown in a layer-by-layer (lbl) fashion on a functionalized substrate. In this synthesis scheme, the reactants, linkers, and nodes are not mixed but are kept apart. Layers of defined thickness $(\sim 5-1,000 \mathrm{~nm}$ thickness, $\sim 1-50 \mathrm{~nm}$ of roughness depending on the MOF system, substrate and growth condition) can then be obtained by alternatingly immersing the functionalized substrate into solutions of inorganic nodes and organic linkers, thus obtaining lbl growth. 
This allows the creation of superstructures with periodicities given by the number of immersion cycles. The thicknesses of individual MOF layers can be easily adjusted using automated techniques, and by varying nodes and linkers between subsequent steps, it is possible to adjust pore sizes and other MOF properties. While the lbl approach allows the vertical structure to be adjusted, different types of lithographic methods can be used for creating lateral features. First, photolithography has been successfully employed to create laterals structures by employing special types of surface-anchored MOF thins films (SURMOFs) [22]. Furthermore, using the microcontact printing method, the substrates used for lbl growth can be functionalized by thiol-based self-assembled monolayers. Since SURMOF growth occurs only on the modified areas, laterally patterned structures can be fabricated. While both lithographies have been demonstrated before [22, 23], they have as yet not been explored in the context of MOF-on-MOF heterostructures.

A schematic view of the hierarchical structures achievable by the $\mathrm{lbl}$ process is shown in Fig. 1 . To realize such a design, the substrate/MOF and the MOF/MOF interface are of crucial importance. As mentioned above, the key difference to the solvothermal synthesis of MOFs is the use of separate solutions of linkers and nodes for the immersion of the functionalized substrate. This approach employing separated educts, also referred to as liquid-phase quasi-epitaxial deposition, was first demonstrated in 2007 [24]. Since then, this particular fabrication method for obtaining MOF thins films, also referred to as SURMOFs $[25,26]$, has been successfully used to construct a variety of MOF-on-MOF heteroepitaxial thin-film structures. One of the unique features of these multilayers is the presence of well-defined heterointerfaces, which act in functional synergy. In this review, we will discuss the design principles of MOF-on-MOF heteroepitaxy, its advantages over contemporary methodologies, and the particular properties of heterostructures and heterointerfaces. We will first focus on lbl methods, which are based on a more straightforward strategy and typically yield planar thin films. In the following section, we will describe different variants of one-pot solvothermal methods, which typically yield core/shell particles. Finally, we will point out emerging applications accessible by hierarchical MOF-on-MOF architectures.

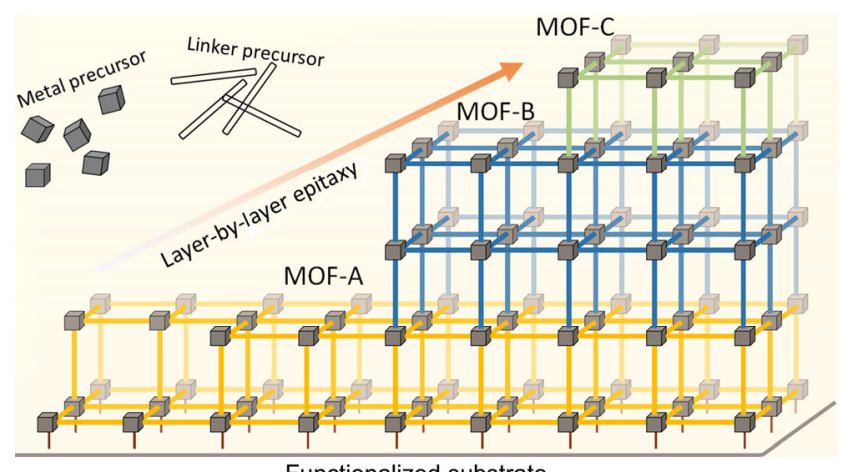

Figure 1 A schematic illustration of the layer-by-layer liquid-phase epitaxy approach to constructing a MOF-on-MOF architecture from a metal and linker precursor. Different colors indicate different organic linkers having a similar length. Instead of a linker, the metal precursor can also be switched during the lbl process to construct a heterometallic architecture.

\section{Epitaxial growth of MOFs and the prospect of heteroepitaxy}

As the first step in conventional solvothermal MOF synthesis, nucleation of crystals takes place, which is a complex process depending on temperature, solvent media, and precursor concentrations. When adding, e.g., a different linker after this nucleation phase, a mixed-linker MOF material (referred to as MOF-on-MOF) can be obtained [27]. Kitagawa and coworkers also used pre-synthesized MOF-A crystals as seeds for MOF-B crystal growth [28]. Nevertheless, MOF-A/B core-shell structures with homogeneous structural properties are difficult to realize. These are elaborately discussed with representative examples in the following Section 4. In addition, many device applications require the deposition of hetero-MOF thin films on flat substrates (e.g., for optical applications). Such architectures are not accessible with the solvothermal MOF-on-MOF approach. Instead, the liquid-phase epitaxy (LPE) method for the growth of SURMOFs in a lbl fashion is a very convenient approach to making MOF-A/B heterostructures.

In the SURMOF approach, metal and linker precursors are deposited in a step-by-step fashion on functionalized substrates (surfaces terminated with $-\mathrm{COOH},-\mathrm{OH},-\mathrm{NH}_{2}$, etc.) $[25,26]$, so that number of layers and the overall thickness can be rationally controlled. Since the different reactants, nodes, and linkers are kept separate, changing the metal or linker precursor after achieving a desired thickness is straightforward. While the size of the individual construction units (unit cells) is adjusted in the isoreticular MOF synthesis approach by choosing appropriate organic linkers, the superstructure periodicity in multi-heterolayer SURMOFs is defined by the number of respective immersion cycles [29]. Benchmark MOF materials, such as HKUST-1 [24], ZIF-8 [30], and UiO-66- $\mathrm{NH}_{2}$ [31], have been successfully grown as SURMOFs in the lbl fashion. In the case of heterostructure construction, metal or linker precursors can be switched without changing any other conditions, such as temperature, solvent, etc. Thus, multilayer MOF structures with controllable layer thickness can be grown maintaining their orientation and crystallinity.

The first demonstration of hetero SURMOFs was published in 2011, when a pillared-layer $\mathrm{Zn} / \mathrm{Cu}$ bilayer heterostructure was fabricated, as shown in Figs. 2(a) and 2(b) [32]. In this case, the basis MOF structure (pillared-layer MOF $[33,34]$ ) consisted of two different linkers, a dicarboxylic acid, 1,4-naphthalene dicarboxylate (ndc), and 1,4-diazabicyclo(2.2.2)octane (dabco). In order to achieve oriented growth of the individual SURMOFs, a pyridine-terminated substrate was used. This surface termination yielded MOF growth along the pillar direction (i.e., dabco axis, see Fig. 2(a)). Whereas the Cu-MOF $\left(\mathrm{Cu}_{2}(\mathrm{ndc})_{2}(\mathrm{dabco}) \sim\right.$ MOF-A) yielded rather well-defined SURMOFs, the structural quality was rather poor for the $\mathrm{Zn}_{2}$ (ndc) ${ }_{2}$ (dabco) (MOF-B) SURMOFs, although the two MOFs are isostructural. The structural quality of the latter could be improved substantially by first growing MOF-A as a bottom layer and then switching the metal source to $\mathrm{Zn}$. A thorough analysis using synchrotronbased X-ray diffractometry revealed that the corresponding hetero-bilayers should perfect the epitaxial growth with high crystallinity and pronounced orientation (Figs. 2(c) and 2(d)). Although the unit cell dimensions of the two MOF structures differed by $\sim 1 \%$, perfect heteroepitaxy was observed.

In the following sections, we will discuss further examples that show the versatility of the heteroepitaxy approach for constructing MOF-on-MOF or MOF-on-other crystalline structures, together with some first applications.

\subsection{Heteroepitaxial MOF-on-MOF thin films: Porosity gradient}

In order to obtain epitaxial growth, one has to consider substrate termination, unit cell dimensions, and symmetry. 
(a)

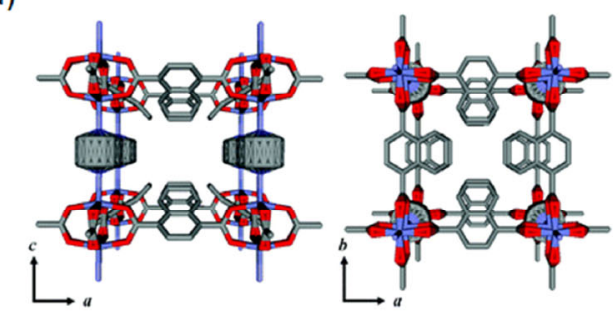

(b)

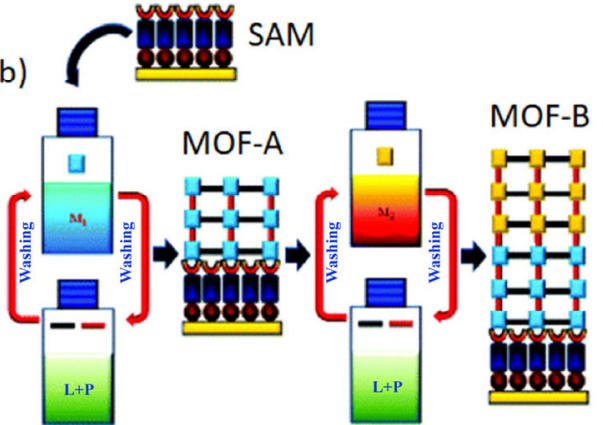

(c)

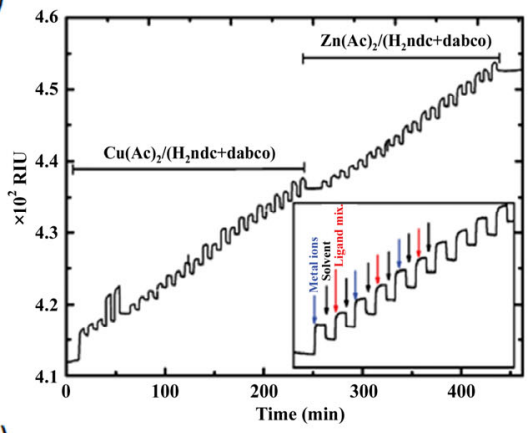

(d)

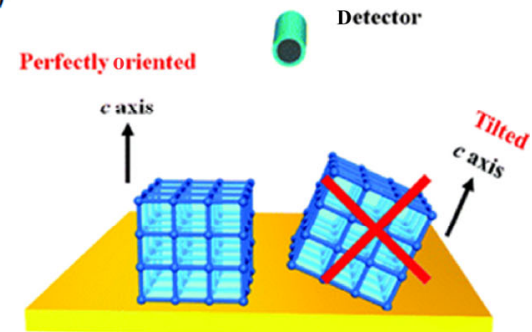

Figure 2 (a) Crystal structures of $\mathrm{Cu}$ - or $\mathrm{Zn}$-based pillared-layer structure $\left[\mathrm{M}_{2}(\mathrm{ndc})_{2}(\mathrm{dabco})\right]$; (b) schematic presentation of the lbl process for growing MOF-A on MOF-B as an epitaxial heterostructure; (c) in situ monitoring of SPR signal during heteroepitaxial growth of MOF-A and MOF-B; inset shows the steps of metal ion and linker addition (RIU = refractive index unit); (d) a schematic view of perfectly oriented crystalline domains (as obtained in the $\mathrm{lbl}$ case) and tilted crystal domains (reproduced with permission from Ref. [32], @ The Royal Society of Chemistry 2011).

With regard to inorganic semiconductors, often a substantial mismatch of symmetry and dimension makes epitaxy impossible; and when aiming at well-defined interfaces, lattice mismatches need to be smaller than $2 \%$, like e.g., in the case of $\mathrm{Pb}$ (111) on Ge (111) [35]. However, in the case of MOFs, their favorable elastic properties, as well as the fairly low energies related to vacancies, have allowed heteroepitaxy even in cases where the lattice mismatch amounted to almost $20 \%$. This example will be discussed below.

MOF-2 is a molecular framework with a two-dimensional (2D) square-net structure that exhibits tetragonal symmetry [36]. This material is polymorphic, and when synthesized with the solvothermal method, a compound with P2 symmetry is obtained. When using the lbl method, a different polymorf of this material is obtained, referred to as SURMOF-2 [29]. The corresponding SURMOFs exhibit $P 4$ symmetry and grow with their [001] orientation perpendicular to the surface. As with other MOFs, SURMOF-2 allows for isoreticular expansion of the $2 \mathrm{D}$ net size, and in the past, numerous SURMOF-2 structures with lattice constants between 1 and $2.9 \mathrm{~nm}$ have been reported [29, 37-41].

Here, we first focus on SURMOF-2 thin layers grown with three different linkers: 1,4-benzenedicarboxylate (bdc), ndc, and biphenyl-4,4'-dicarboxylic acid (bpdc) (see Fig. 3(a)). The $\mathrm{X}$-ray diffraction (XRD) data shown in Fig. 3(a) reveal good crystalline order in all three cases, with lattice constants of $1.12,1.34$, and $1.55 \mathrm{~nm}$. In all cases, the thin films are perfectly orientated. The difference in length of the bpdc linkers and the ndc linkers amounts to $\sim 20 \%$. As noted above, in the case of "hard" inorganic materials, such a large lattice constant mismatch would make the epitaxial growth of these compounds on substrates made from the other compound impossible. However, in the SURMOF-2 case, as demonstrated by the data in Fig 3(a) with a second layer grown on top of a different first layer, excellent crystallinity and a high degree of orientation is observed in both cases, i.e., growing the SURMOF with the longer lattice constant on top of the one with the smaller unit cell and vice versa [42]. As demonstrated by a thorough theoretical analysis using accurate force-constant fields, the intrinsic elasticity of MOF crystals strongly reduces the energy penalty in the case of lattice mismatch.

A staining technique was employed to unequivocally demonstrate the presence of vertically stacked hetero-multilayers with well-defined interfaces between them. To this end, samples were immersed in a solution of a metal-organic compound, $\mathrm{Eu}(\text { bzac })_{3}$ bipy $\left(\right.$ bzac $=1$-benzoylacetone, bipy $=2,2^{\prime}$-bipyridine $)$. The size of this guest amounts to $0.97 \mathrm{~nm}$, so only the largest pore size Cu-bpdc SURMOF-2 (pore size of $1.2 \mathrm{~nm} \times 1.2 \mathrm{~nm}$ ) could accommodate this guest compound, while the other two smaller pore sizes exclude the guest. Indeed, the size-selective encapsulation of the Eu complex in the bottom layer of a trilayer $\mathrm{Cu}-\mathrm{bpdc} / \mathrm{Cu}-\mathrm{ndc} / \mathrm{Cu}$-bdc heterostructure was evident from the scanning electron microscopy (SEM) cross-sectional image shown in Fig. 3(b). To estimate the interface defect concentration, a Cu-trifluoroacetate precursor was used to estimate the concentration of metal sites not integrated in the MOF lattice by recording the intensity of the C-F stretch vibration. The rather small intensity of this band was found to be in accordance with the structural model of the heterointerface derived from theoretical work, which also demonstrated rather low strain at the boundaries between the two different MOF types [42].

Later, Fischer and coworkers demonstrated that heteroepitaxial growth of lattice mismatched SURMOFs of different symmetry [43]. To construct such heterostructures, the outer (upper) surface of a SURMOF was selectively modified so it acted as a templating substrate for the oriented growth of a different MOF type. For this demonstration, first a supporting SURMOF of the type HKUST-1 or $\mathrm{Cu}_{3} \mathrm{btc}_{2}$ (btc $=1,3,5$-benzenetricarboxylate) was grown. In the final step in the lbl process employed to grow this MOF thin film, the btc linker was replaced by a modified btc linker (fu-ip, or functionalized isophthalic acid) having a functional group (pyridine (py), hydroxy $(\mathrm{OH})$, butoxy $(\mathrm{Bu})$, and 4 -hydroxybutoxy $(\mathrm{BuOH}))$ at the $5^{\text {th }}$ position instead of carboxylic acid (Fig. 4). The use of functionalized isophthalic acid restricted the further growth of HKUST-1 on top. These functionalized MOF layers were then used to grow a secondary three-dimensional (3D) MOF $\mathrm{Cu}_{2} \mathrm{ndc}_{2}$ dabco in 
(a)
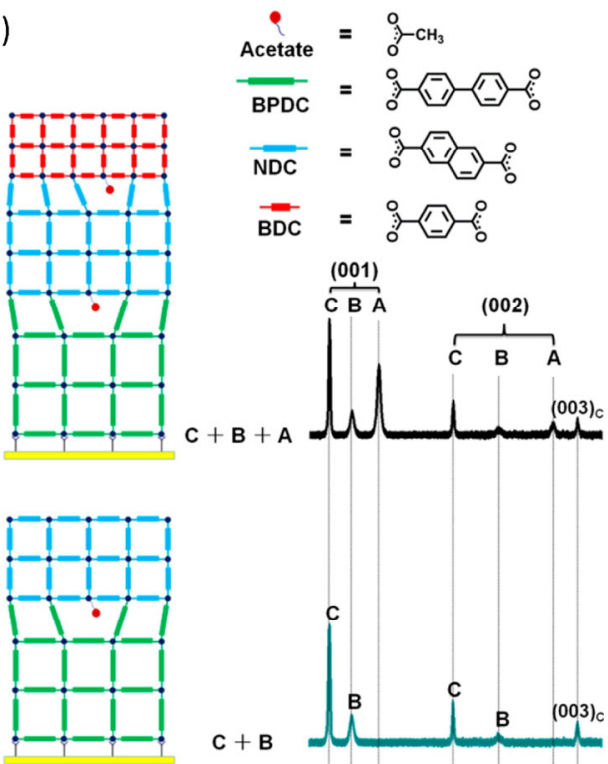

$\mathrm{C}+\mathrm{B}$

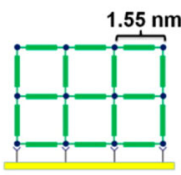

MOF-C
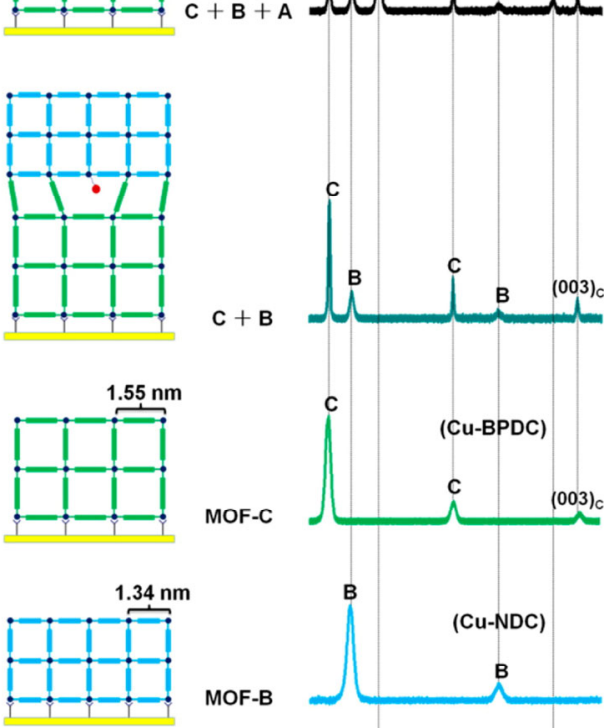

MOF-B
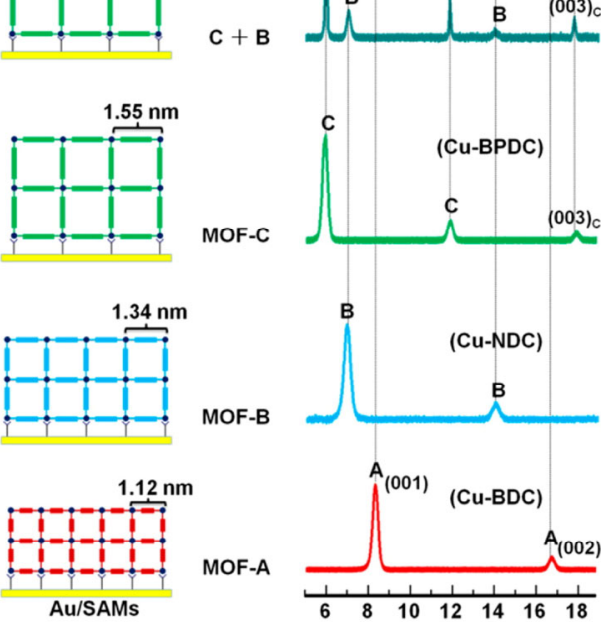

MOF-A

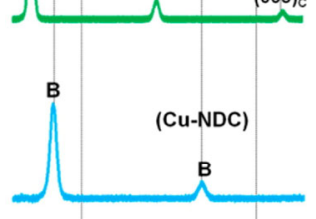

(b)

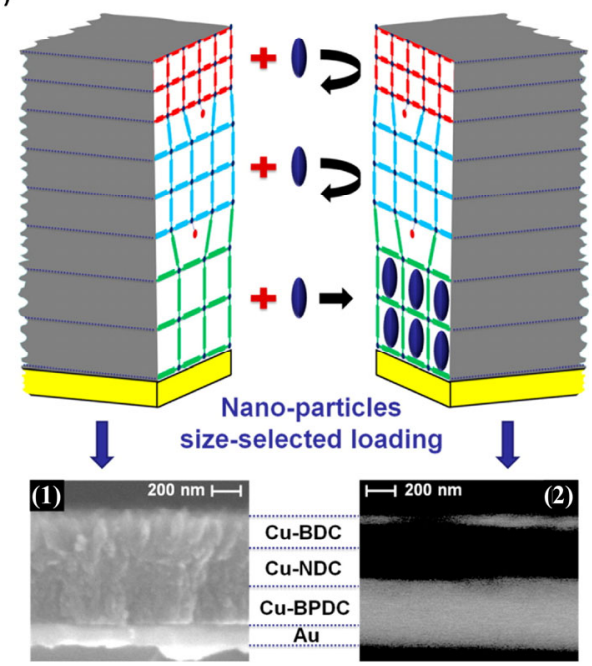

Figure 3 (a) Left: Schematic structures of the hierarchically porous MOFs $(\mathrm{Cu}-\mathrm{bpdc}+\mathrm{Cu}-\mathrm{ndc}+\mathrm{Cu}-\mathrm{bdc})$ with large lattice mismatches grown on selfassembled monolayer-modified $\mathrm{Au}$ substrates using the layer-by-layer LPE method (dark blue circles represent metal connectors). Upper right: Molecular structures of the organic linkers. Lower right: Out-of-plane XRD data recorded for the SURMOFs illustrated on the left. (b) Top: Schematic of nanoparticle size-selective loading in hierarchically porous SURMOFs. Bottom: (1) SEM image recorded for a hierarchically porous SURMOF (Cu-bpdc + Cu-ndc + Cu-bdc) with $5 \mathrm{~nm}$ gold films coated on the surface. (2) SEM image recorded for a hierarchically porous SURMOF $(\mathrm{Cu}-\mathrm{bpdc}+\mathrm{Cu}-\mathrm{ndc}+\mathrm{Cu}-\mathrm{bdc})$ after loading Eu(bzac) $)_{3}$ bipy without coating gold films on the surface. Reproduced with permission from Ref. [42], (c) American Chemical Society 2014.

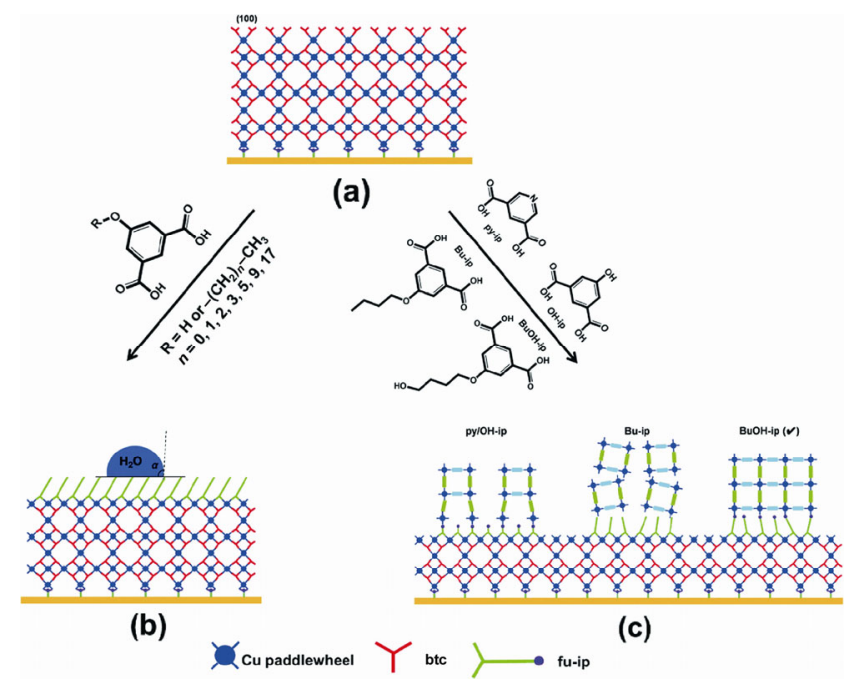

Figure 4 Schematic view of (a) HKUST-1 SURMOF along the [100] direction anchored to carboxylate-functionalized Au-coated quartz crystal microbalance substrate; (b) implementation of fu-ip ligands (consisting of hydrophobic functional groups at the $5^{\text {th }}$ position) on the external surface of HKUST-1; (c) hetero-growth of $\mathrm{Cu}_{2} \mathrm{ndc}_{2}$ dabco on the top of HKUST-1 after functionalizing the interface with different kinds of fu-ip ligands (blue: $\mathrm{Cu}$ paddlewheel, red: btc linker, and green and violet: fu-ip ligand) (reproduced with permission from Ref. [43], (c) The Royal Society of Chemistry 2018).

epitaxial fashion. Measurements using out-of-plane XRD demonstrated that, for $-\mathrm{BuOH}$ functionalization, the growth of a secondary SURMOF was successful, thus yielding a hetero-bilayer SURMOF. Flexibility of the $\mathrm{BuOH}$ functional group was considered a deciding factor in the secondary nucleation of MOF crystals.

Fischer and coworkers have demonstrated the heterostructure of $\mathrm{Cu}_{3}(\mathrm{btc})_{2}$-on- $\mathrm{Cu}_{2}(\mathrm{ndc})_{2}(\mathrm{dabco})$, having cubic and tetragonal symmetry, respectively [44]. In this work, first $\mathrm{Cu}_{2}(\mathrm{ndc})_{2}(\mathrm{dabco})$ pillared-layer MOF was grown on substrates functionalized with pyridine-terminated self-assembled monolayers with the crystallographic [001] direction (i.e., pillar dabco) perpendicular to the surface. In the last step of growth, outer surface was terminated with dabco pillars. This dabco-terminated surface was used as a substrate for the growth of $\mathrm{Cu}_{3}(\mathrm{btc})_{2} \mathrm{SURMOF}$, which was found to grow along the [111] direction. Interestingly, it was found that the inverted system, i.e. growth of $\mathrm{Cu}_{2}(\mathrm{ndc})_{2}$ (dabco) SURMOFs on $\mathrm{Cu}_{3}(\mathrm{btc})_{2}$ substrates, could not be realized. This is because the outer surface of $\mathrm{Cu}_{3}(\mathrm{btc})_{2}$ MOF contains tilted carboxylate groups (i.e. btc linker) which cannot template the growth of $\mathrm{Cu}_{2}(\mathrm{ndc})_{2}$ (dabco) MOF along [001] direction.

A rather appealing application of hetero-bilayer architectures is to construct controlled release systems that consist of a top valve placed on a bottom container. Indeed, a photoresponsive construct for controlled release of guest molecules has been realized using the SURMOF approach. To this end, a heterobilayer was fabricated with the bottom layer assembled from $\mathrm{Cu}_{2}(\mathrm{bpdc})_{2}$ (bipy) and a top layer grown using linkers containing photoswitchable azobenzene side groups, $\mathrm{Cu}_{2}(\mathrm{ab}-\mathrm{bpdc})_{2}$ (bipy) (ab-bpdc: 2-azobenzene 4,4'-biphenyldicarboxylic acid) (Fig. 5(a)) [45]. As azobenzene can be switched between cis and trans forms by irradiating with UV (trans $\rightarrow$ cis) and visible (cis $\rightarrow$ trans) light, the controlled release of guest from the porous bottom MOF structure is feasible. The performance of the corresponding device was demonstrated for butanediol, where the uptake was $\sim 15$ times faster for the azobenzene groups in the top SURMOF when switched to trans rather than when switched to cis form. Also, the release observed after loading could be efficiently controlled by irradiation with light (Figs. 5(b) and 5(c)). 


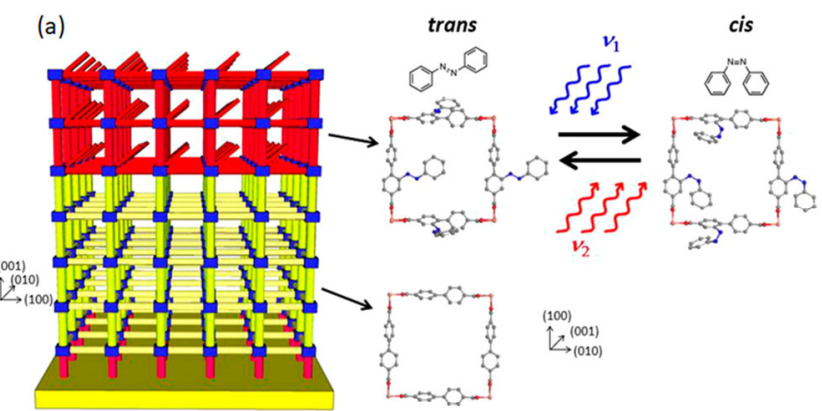

(b)

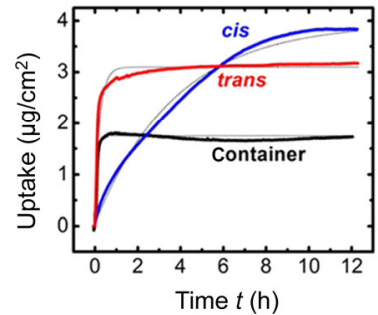

(c)

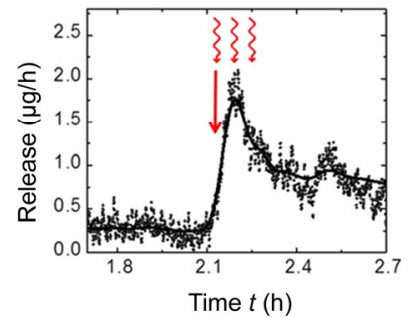

Figure 5 (a) Structural drawing of heteroepitaxial two-component MOF: the $\mathrm{Cu}_{2}(\mathrm{bpdc})_{2}$ (bipy) bottom layer (yellow) acts as a container for storing molecules, and the photoswitchable $\mathrm{Cu}_{2}(\mathrm{ab}-\mathrm{bpdc})_{2}$ (bipy) top layer (red) acts as a gate. Reversible switching occurs from trans to cis by UV light $\left(v_{1}\right)$ and from cis to trans by visible light $\left(v_{2}\right)$. (b) Butanediol uptake by the bilayered SURMOF in the trans state (black) and in the cis state (violet). The time constants of the monoexponential fits (thin dotted lines) are $0.23 \mathrm{~h}$ in the trans and $3.6 \mathrm{~h}$ in the cis state. The uptake by pristine $\mathrm{Cu}_{2}(\mathrm{bpdc})_{2}$ (bipy) SURMOF is plotted in gray (with a time constant of $0.06 \mathrm{~h}$ ). (c) Release of the guest molecule from the bilayer photoswitchable SURMOF determined by quartz crystal microbalance. Red arrow indicates irradiation with $560 \mathrm{~nm}$ wavelength light, initiating the release. Reproduced with permission from Ref. [45], () American Chemical Society 2014.

Another example of MOF-on-MOF heterostructures for tuning the affinity towards analyte (volatile organic compounds) was reported by Kitagawa, Fischer, and coworkers [46]. In this work, using LPE deposition followed by post-synthetic modification, two heterostructures were fabricated on $\mathrm{Au}$-coated quartz crystal sensors. Two different, inverse deposition sequences were used; in type-I MOF-C@MOF-A and type-II MOFA@MOF-C (MOF-A = Cu (ndc) $_{2}$ dabco; MOF-C $=\mathrm{Cu}_{2}$ (HOOC$\left.\left(\mathrm{CH}_{2}\right)_{2} \mathrm{OCNH}-\mathrm{bdc}\right)_{2}$ dabco $)$. In this Janus type MOF heterostructures, MOF-A is nonpolar and MOF-C acts as polar container In the vapor adsorption experiments, it was revealed that the small polar analyte methanol was adsorbed in equal volume by both types of heterostructures. However, in case of the nonpolar, slightly larger hexane, type-I heterostructures did not show any adsorption, while type-II heterostructure adsorbed 0.5 molecule hexane/Cu-paddle-wheel unit of the MOF. This was explained by the fact that in type-I the top MOF layer is polar and exhibits a small pore size only. Mixture of methanol/ hexane vapor adsorption experiment also exhibited a selective permeation of methanol over hexane in type -I heterostructures. This result strongly indicates that MOF-on-MOF assemblies realized by the LPE method are rather effective for designing heterogeneous crystalline materials with pronounced synergy effects in the context of gas separation.

In a recent example of MOF-on-MOF hierarchical structure fabrication, Takahashi et al. demonstrated that SURMOFs of type SURMOF-2 can be grown so they yield a preferential azimuthal orientation, with a homogeneous orientation over macroscopic dimensions [47]. This case used a particular substrate functionalization employing an azimuthally oriented $\mathrm{Cu}(\mathrm{OH})_{2}$ nanobelt as a sacrificial support. Upon immersion of such substrates into a linker (bdc) solution, the epitaxial growth of $\mathrm{Cu}$ (bdc) SURMOF-2 was observed [48]. Using the same approach, a $\mathrm{Cu}(\mathrm{bpdc})$ SURMOF-2 layer was grown successfully. As a secondary layer, $\mathrm{Cu}(\mathrm{bdc})$ SURMOF-2, an isoreticular structure to $\mathrm{Cu}(\mathrm{bpdc})$ was grown in a lbl fashion using the LPE method (Fig. 6). In-plane as well as out-ofplane and azimuthal angle-dependent XRD studies revealed perfectly oriented MOFs and identical in-plane orientation of the $\mathrm{Cu}(\mathrm{bdc})$ and $\mathrm{Cu}(\mathrm{bpdc})$ structures. The authors of this study were also able to grow a third heterolayer consisting of $\mathrm{Cu}(\mathrm{ndc})$ SURMOF-2 on top of this bilayer system, thus yielding a fully aligned, heteroepitaxial trilayer system. We would like to point out that such hetero-multilayers are accessible only via the LPE approach.

\subsection{Heteroepitaxial MOF-on-MOF thin films: Photophy- sical properties}

As discussed in the Introduction, a unique feature of MOF hetero-multilayers are well-defined heterojunctions between different metal-organic frameworks. In some applications, such highly ordered interfaces have interesting properties, e.g., in the case of charge separation in organic photovoltaics or optical properties, e.g. photon upconversion.

In the case of upconversion, the most direct approach is to mix donors and acceptors to accomplish the transfer of triplet-excitons from a donor (e.g. a porphyrin) to an acceptor (e.g. anthracene), where triplet-triplet annihilation occurs. Such mixtures, however, are typically amorphous, containing a large density of structural imperfections. As a result, diffusionenhanced non-radiative decay of triplet excitons is the major limiting factor in these materials [49]. The realization of crystalline, heteroepitaxial donor/acceptor multilayers with well-defined interfaces across which exciton transfer can occur efficiently thus offers an important improvement.

Howard et al. employed the lbl-based multiheteroepitaxy approach to build an ABA type of SURMOF heterostructure. In this case, two different chromophoric linkers were used to realize the two different MOF types: a Pd(II)porphyrin linker (type A) and an anthracene-based linker (B). Although there

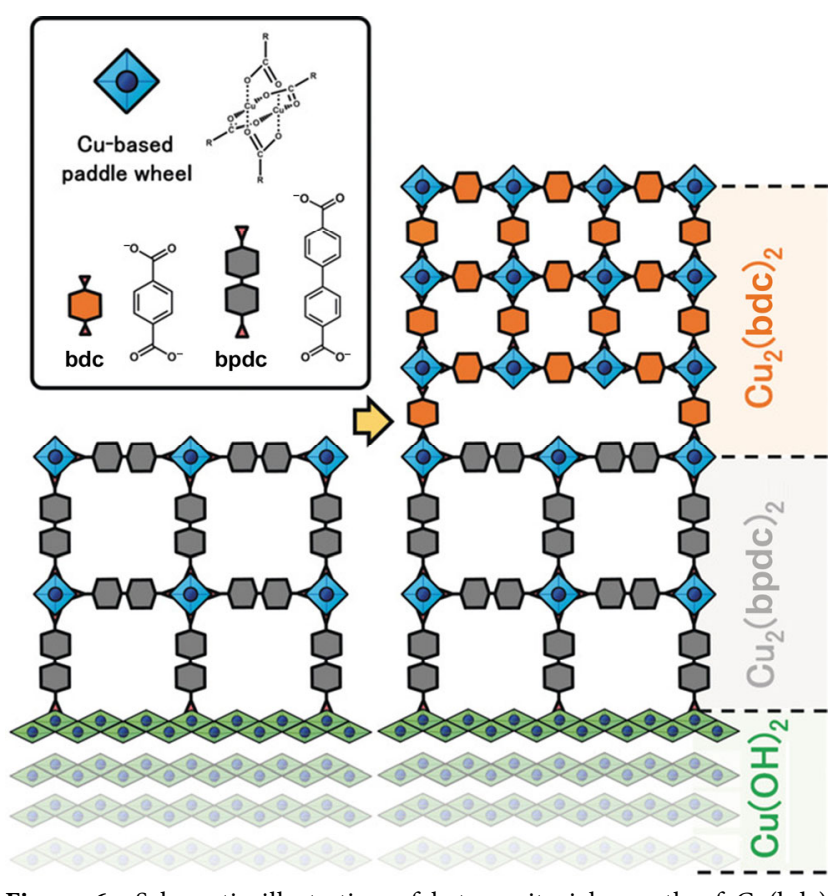

Figure 6 Schematic illustration of heteroepitaxial growth of $\mathrm{Cu}(\mathrm{bdc})$ SURMOF-2 on $\mathrm{Cu}(\mathrm{bpdc})$ SURMOF-2 using the LPE method on in-planeoriented $\mathrm{Cu}(\mathrm{OH})_{2}$ support layer (reproduced with permission from Ref. [47], (c) Wiley-VCH Verlag GmbH \& Co. KGaA, Weinheim 2019). 
is a small lattice mismatch between the corresponding two different SURMOF-2 systems, about 5\%, structurally welldefined bilayers of type $\mathrm{AB}$ and $\mathrm{BA}$ as well as $\mathrm{ABA}$ heterolayers could be grown (see Figs. 7(a) and 7(b)). These systems were then used successfully to demonstrate photon upconversion. In this process, first the efficient light absorption and subsequent singlet-triplet conversion of the porphyrin-based linkers were used to create a high density of triplet excitons created in the A-type SURMOFs. When these triplet excitons reach the $\mathrm{AB}$ interface by diffusion, the excitons can be transferred, via a Dexter mechanism, into the anthracene-based B SURMOFs. The latter show a high yield for triplet-triplet annihilation, yielding an overall photon upconversion of green photons into blue photons (Fig. 7(c)) [50]. The upconversion threshold was found to be $\sim 1 \mathrm{~mW} / \mathrm{cm}^{2}$.

The availability of organic/organic interfaces of high structural quality also increased the insight into exciton transport in crystalline chromophore assemblies. The anisotropic nature of exciton diffusion in such systems was demonstrated for a SURMOF-2 type donor/acceptor bilayer fabricated using appropriate chromophoric linkers, as shown in Figs. 8(a) and 8(b) [51]. In this case, the bottom SURMOF was made from diketopyrrolopyrrol (dpp) chromophores, acting as energy acceptors, whereas the crystalline MOF thin film grown epitaxially on top was assembled from anthracenedibenzoate (adb) linkers acting as energy donors. In this heterostructure, the lattice mismatch between the two different SURMOFs was small ( 3\%), and hence, well-defined crystalline interfaces could be obtained. After excitation of the chromophores in the top adb layer by illumination with $400 \mathrm{~nm}$ light, the excitons can diffuse either along the (010) direction within the highly anisotropic adb chromophore layer or perpendicular to the layer ((001) or (100) directions). Since reaching the interface to the bottom dpp layer and then creating an excitation of dpp chromophores require diffusion in the vertical direction, the ratio of vertical and parallel diffusion lengths within the adb layer can be determined from time-resolved fluorescence measurements recorded for bilayer systems with different thicknesses of the top layer (adb layer). A careful analysis reveals that exciton diffusion is highly anisotropic and much more efficient in a direction parallel to the substrate plane, i.e., along the (010) axis.

SURMOF multilayers obtained by stacking layers grown with different porphyrinic linkers were also investigated recently [52]. It was shown that a combination of three porphyrinbased SURMOF heterolayers can efficiently absorb light across the entire visible range of the solar spectrum.
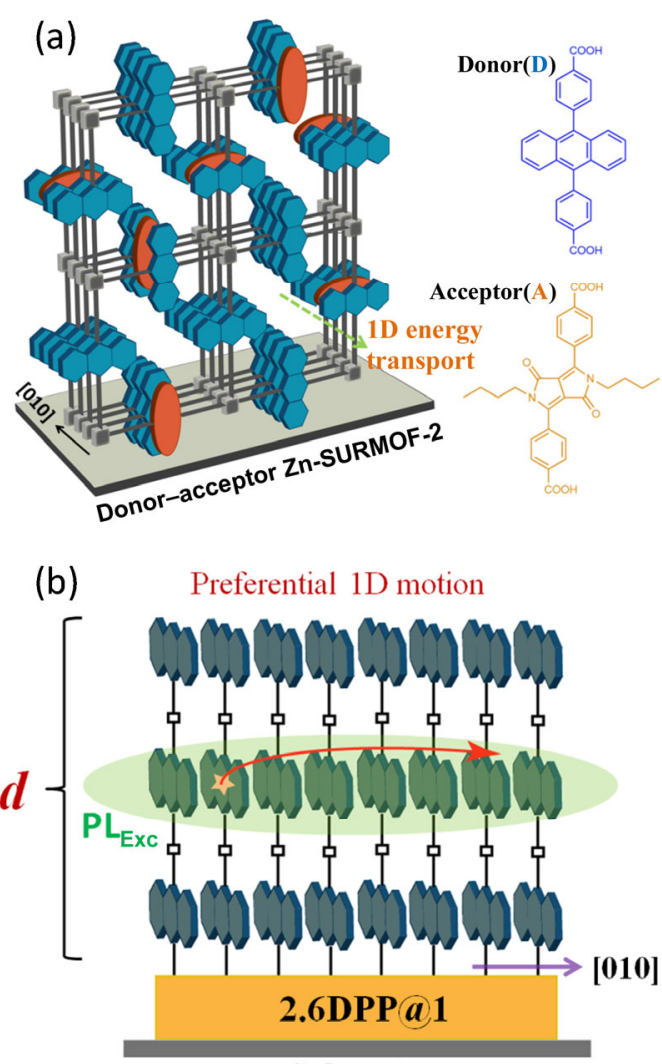

Substrate

Figure 8 (a) Schematic illustration of a donor-acceptor Zn-SURMOF-2 structure showing the preferred exciton diffusion path along the (010) direction. (b) Heteroepitaxial Zn-SURMOF-2 structure in which the preferential motion of exciton (PLExc) is shown. Reproduced with permission from Ref. [51], (c) Haldar, R. et al. 2018.

The special opportunities of SURMOF bilayer systems fabricated using a lbl approach have also been used by Hupp and coworkers to demonstrate directional energy transport in chromophoric assemblies [53]. Their study was based on the isoreticular series of pillared-layer Zn-MOFs, containing 1,2,4,5tetrakis(4-carboxyphenyl)benzene (L1) linker and different types of pillar linkers (energy donor-acceptor), $N, N^{\prime}$-di $(4-$ pyridyl)-1,6,7,12-tetrachloro-3,4,9,10-perylenetetracarboxylic diimide $\left(\mathrm{PDICl}_{4}\right)$ and $N, N^{\prime}$-di(4-pyridyl)-1,7-di(3,5-di-tertbutylphenoxy)-3,4,9,10-perylenetetracarboxylic diimide $\left(\mathrm{PDIOPh}_{2}\right)$. Using polarization-dependent absorption experiments, it was confirmed that the pillared-layer structure (a)

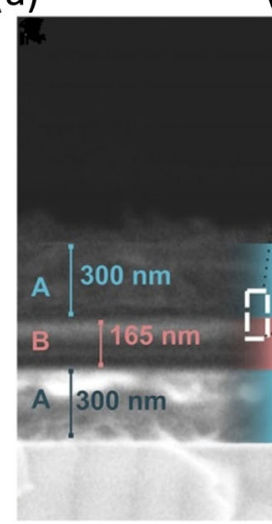

(b)

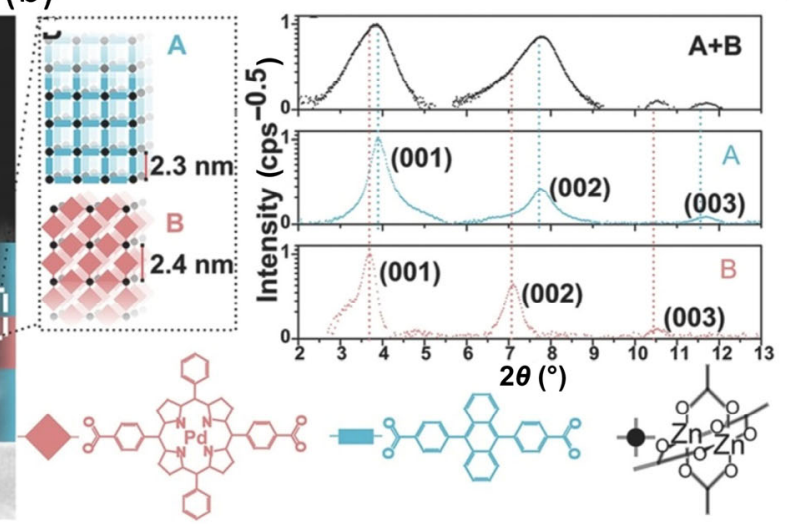

(c)

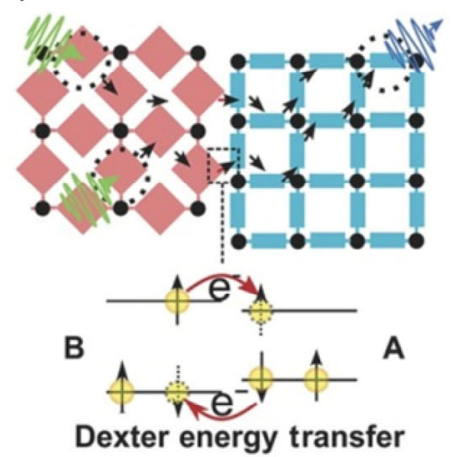

Figure 7 (a) View of a trilayer heteroepitaxial SURMOF-2 constructed of Pd-porphyrin and anthracenedibenzoate linkers from cross-section scanning electron microscopy image. (b) Out-of-plane XRD data of the trilayer and pristine SURMOF-2 structures. (c) Illustration of the Dexter energy transfer at the organic-organic interface of the trilayer SURMOF. Reproduced with permission from Ref. [50], () Wiley-VCH Verlag GmbH \& Co. KGaA, Weinheim 2016. 
grows along the pillar direction, resulting in vertically aligned transition dipoles of the pillars. This facilitated the Förster-type energy transfer in the vertical direction. Deposition of a dye acceptor on top of the bilayer structure resulted in complete energy transfer from bottom MOF to top layer. A similar layered heterostructure design can promote an understanding of interfacial photophysics, which is fundamental to photovoltaics and related optoelectronic applications.

In these systems discussed above, the difference in the photophysical properties of the components in hetero SURMOFs resulted from using different types of linkers; however, the nodes, consisting of metal or metal-oxo clusters, can also be employed. In this context, employing lanthanide (Ln)-based nodes is particularly attractive, since the chemistry of these metal ions is very similar. Accordingly, one would expect that the structural parameters of a Ln-based MOF would vary only a little when using a different lanthanide. Redel and coworkers recently demonstrated that, indeed, this approach enables the heteroepitaxy of Ln-based SURMOFs to be realized in a straightforward fashion [54].

With regard to obtaining materials in which the emission color can be straightforwardly tuned, heterometal MOFs obtained by simple mixing of $\mathrm{Eu}(\mathrm{III}) / \mathrm{Tb}$ (III) for the solvothermal MOF syntheses have been a popular choice [55]. However, in such random mixtures, the very efficient energy transfer (FRET) between neighboring $\mathrm{Tb}(\mathrm{III})$ to $\mathrm{Eu}(\mathrm{III})$ centers causes unwanted quenching effects and severely complicates the color tuning [56]. Here, hetero-bilayers, where a SURMOF made from only one lanthanide is stacked on top of a second SURMOF made from the second lanthanide ion, have a striking advantage. This is because the FRET transfer is only short range and thus basically limited to the heterointerface. This was demonstrated for the case of MOF-76 made from $\mathrm{Tb}$ (III)/Eu(III) nodes and btc linkers. Chen et al. demonstrated that both lanthanide MOF-76 SURMOFs (i.e., Eu(III) and Tb(III)) could be grown with high structural quality using the lbl method (Fig. 9(a)). In a second step, a hetero SURMOF was deposited on top. In this case, the lattice constant difference amounted to less than $1 \%$, and lattice mismatch therefore was not a problem. A careful analysis of the result revealed that, in the heteroSURMOF case, the unwanted quenching effects discussed above are absent-It is straightforward to tune the emission color by simply varying the thickness of the individual layers (Figs. 9(b) and $9(\mathrm{c})$ ). The linear dependence of the emission color change with the top layer thickness allows for precise color tuning and also supports the advantages of the crystalline interface by the LPE approach (Fig. 9(c)).

\section{Multilayer MOF hybrids fabricated by the}

\section{LBL approach}

In addition to MOF-on-MOF multilayers, the lbl approach can be used to create hetero-multilayers containing other materials, e.g., oxides or metals. This has been demonstrated by Liu et al., who combined lbl deposition with sputter deposition of an oxide to fabricate one-dimensional (1D) photonic crystals exhibiting a photonic bandgap (PBG) (Fig. 10) [57]. Such a Bragg stack of HKUST-1 SURMOF and indium tin oxide (ITO)

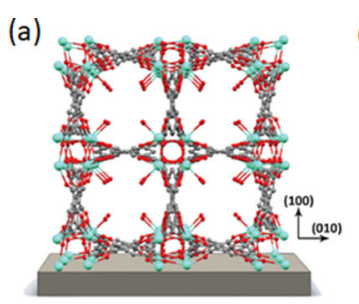

(c)

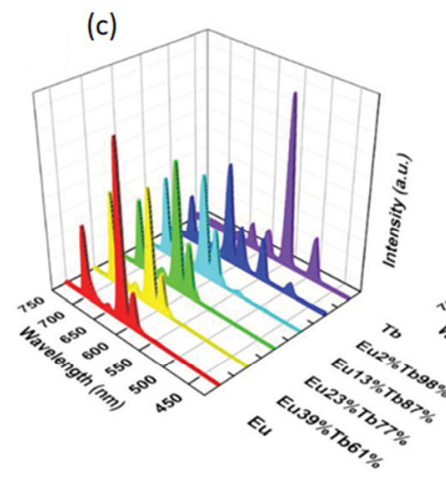

(e)

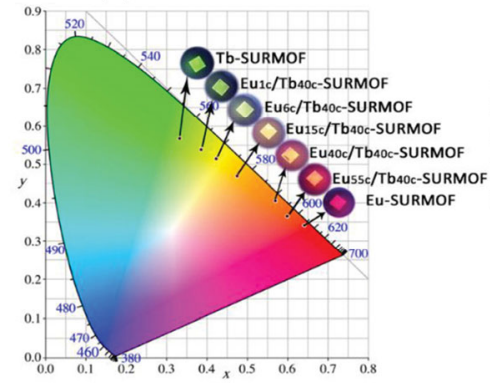

(b)

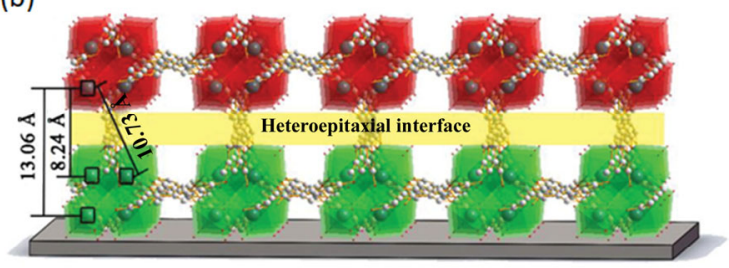

(d)

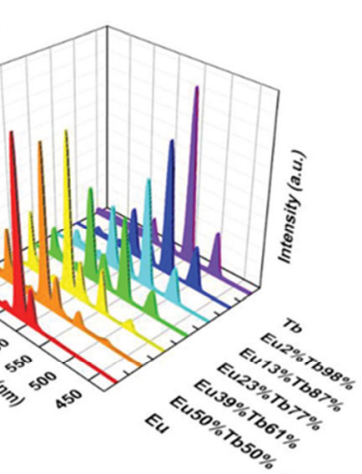

(f)

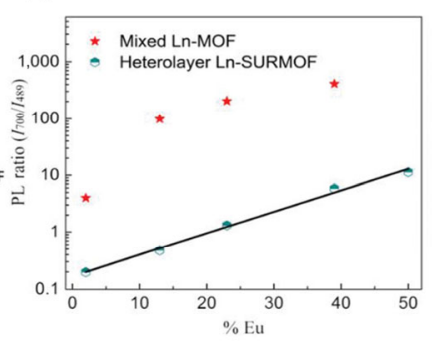

Figure 9 (a) Structure of Tb/Eu-btc SURMOF (MOF-76). (b) View of the heteroepitaxial interface of Tb-Eu-btc bilayer SURMOF. (c) and (d) Emission spectra of Ln-SURMOFs fabricated by doping Eu/Tb ions in the same layer and as a heteroepitaxial bilayer. (e) CIE chromaticity diagram showing the fluorescence color of Eu/Tb-SURMOFs. (f) Ratio between the intensity of the $700 \mathrm{~nm}$ peak (Eu) and the intensity of the $489 \mathrm{~nm}$ peak (Tb) in mixed and heterolayer Eu/Tb-SURMOFs. Reproduced with permission from Ref. [54], (c) Wiley-VCH Verlag GmbH \& Co. KGaA, Weinheim 2019. 
(a)

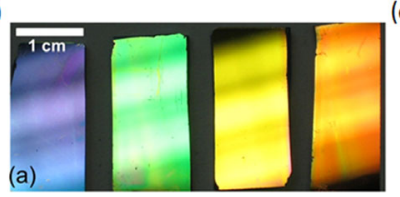

(b)

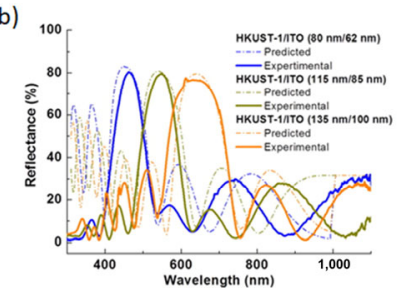

(c)

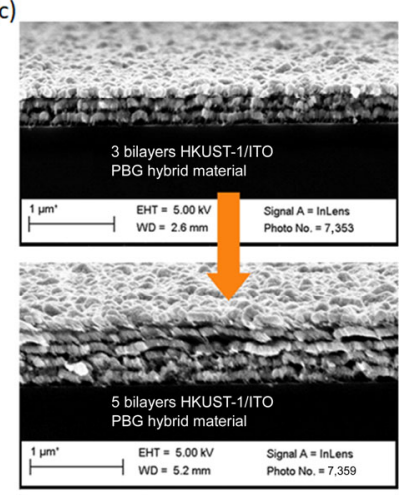

Figure 10 (a) Photonic bandgap SURMOF/ITO materials with different thicknesses. (b) Calculated and experimental reflectance of a 5-bilayer PBG consisting of HKUST-1/ITO ranging from 80 to $135 \mathrm{~nm}$ and from 62 to $100 \mathrm{~nm}$, respectively. (c) High-resolution SEM (HR-SEM) cross sections of multilayered 3-bilayer and 5-bilayer HKUST-1/ITO materials. Reproduced with permission from Ref. [57], () American Chemical Society 2015.

was prepared by alternately applying the $\mathrm{lbl}$ and sputtering processes, respectively, to obtain the desired number of stacked layers. As a result of the deposition process, the surface of the sputter-deposited ITO layers contained a large number of $-\mathrm{OH}$ functionalities. As a result, they were well suited to serve as a templating layer for the subsequent $\mathrm{lbl}$ deposition of HKUST-1 SURMOFs. The two materials are highly suitable for building photonic bandgap materials, since the optical constant of HKSUT-I is rather low (refractive index 1.5), whereas that of ITO is fairly large with a refractive index of $\sim$ 2. In fact, a HKUST-1/ITO stack deposited on a Si substrate containing 10 alternate layers of the two materials showed a reflectance of around $\sim 80 \%$ at the optimum PBG wavelength (650 nm), indicating outstanding optical quality with low scattering loss from the multilayer system. In addition to the interesting optical properties of these hetero-multilayers, the intrinsic porosity of the HKUST-1 layer enabled the straightforward use of the Bragg stacks as optical sensors. Upon loading

with guest molecules (ethanol, isopropanol, tetrahydrofuran, cyclohexane, toluene), the optical constant of the MOF layers changed slightly. It was observed that the optical reflection peak was shifted by $20-40 \mathrm{~nm}$, depending on the nature of the guest molecule loaded into the porous PBG material. Later, Heinke and coworkers used a similar approach to fabricate photoswitchable $\mathrm{MOF} / \mathrm{TiO}_{2}$ Bragg stacks [58]. In this case, additional functionality was added to the bandgap material by employing a photoresponsive azo functional group. Switching the azo groups from trans to cis causes small changes in the optical constant of the MOF systems. As a consequence, the reflectance maximum of the Bragg stack of $\mathrm{MOF} / \mathrm{TiO}_{2}$ could be reversibly shifted between two different positions by illumination with UV and visible light.

\section{Solvothermal syntheses strategies for MOF-}

\section{on-MOF architectures}

In this section strategies to obtain MOF-on-MOF growth are described, which are quite different from those described in the previous section. In addition to the different methodology also the targeted application fields are quite dissimilar. We will discuss several cases, mainly based on solvothermal approaches. As mentioned before, the first demonstration of MOF-onMOF growth occurred using the conventional method of MOF synthesis early in 2009 by Kitagawa and coworkers [28]. By combining $\mathrm{Zn}(\mathrm{II})$ - and $\mathrm{Cu}(\mathrm{II})$-based nodes, ndc linker, and dabco pillar, they were able to demonstrate that layers of the isostructural $\mathrm{Zn}$-containing MOF could be grown on the $\mathrm{Cu}$-containing MOF particles. It was observed that the $\mathrm{Zn}$ based MOF structure grows as a perfect single crystal of few hundred-microns size, but $\mathrm{Cu}-\mathrm{MOF}$ formed in a polycrystalline powder forms. Using Zn-MOF particles as a seed crystal in a solvothermal reaction, it was possible to grow $\mathrm{Cu} / \mathrm{Zn}-\mathrm{MOF}$ core-shell structures (Fig. 11(a)). A detailed analysis of the $\mathrm{XRD}$ data revealed the single crystalline nature of the core and shell structures and confirmed that $\mathrm{Cu}-\mathrm{MOF}$ was grown

(a)
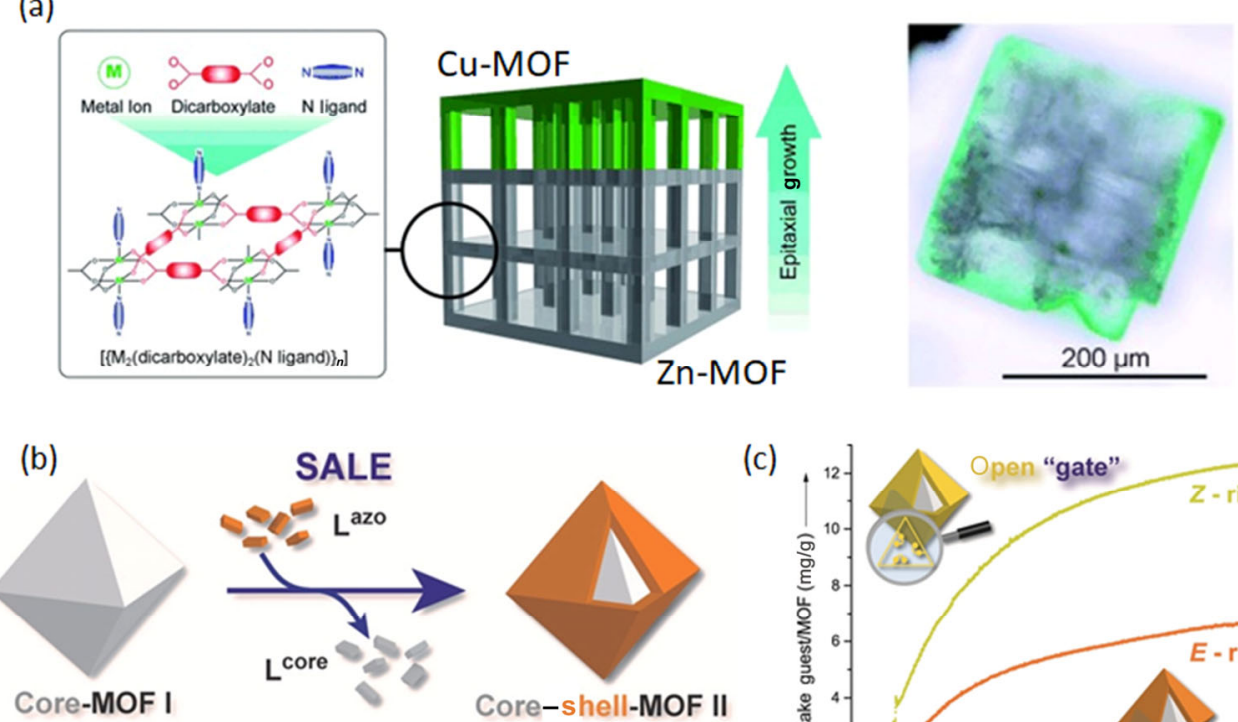

(c)

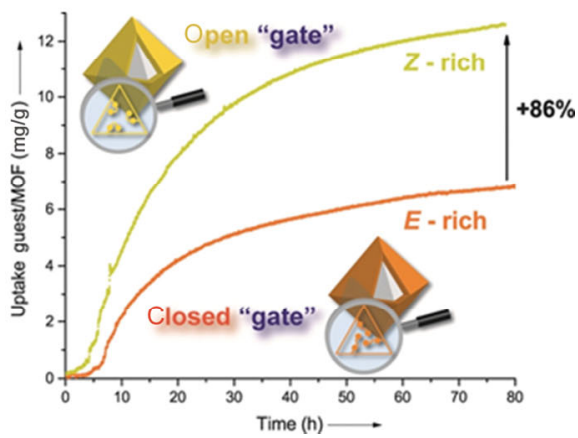

Figure 11 (a) Epitaxial growth of isoreticular Cu-MOF on Zn-MOF as a core-shell structure; (right) optical image of Zn-MOF-Cu-MOF core-shell structure (reproduced with permission from Ref. [28], ๔ Wiley-VCH Verlag GmbH \& Co. KGaA, Weinheim 2009). (b) Schematic illustration of porous core-photoswitchable shell design of MOF-on-MOF architecture by SALE method. (c) Photoswitching guest uptake capacity of core-shell MOF. Reproduced with permission from Ref. [61], @ Wiley-VCH Verlag GmbH \& Co. KGaA, Weinheim 2019. 
epitaxially with an in-plane (dicarboxylate 2D layer plane) rotational angle.

In the same year, Kitagawa and coworkers demonstrated the face-selective epitaxial growth of a second MOF on crystals of a first type of MOF, again using pillared-layer tetragonal symmetry types of MOFs with different length of pillars [59]. The two individual MOFs have different pillar lengths (dabco 9.611(1) $\AA$ and N,N'-di(4-pyridyl)-1,4,5,8-naphthalenetetracarboxydiimide (dpndi, 44.456(4) $\AA$ ) and exhibit almost identical 2D layer lattice dimensions (along the $a$ or $b$ axes). Again, a solvothermal synthesis scheme was used. First, crystals of the dabco-based MOF were grown, which were then used as a seed for dpndi-based MOFs. Finally, again the dabco-based MOF was grown, yielding BAB-type, block-shaped crystals. An X-ray diffraction measurement of the $\mathrm{BAB}$ crystal confirmed the epitaxial growth of the heterostructure.

One advantage of core-shell MOF-on-MOF materials over powders consisting of single-type MOF particles is the improved selective $\mathrm{CO}_{2}$ uptake over $\mathrm{N}_{2}$. This was elegantly demonstrated by Rosi et al. in 2013 using the isoreticular series of a bio-MOF, i.e., a MOF where biomolecules are used as linker components [60]. A porous core structure of a bio-MOF-11/14 mixture and a shell structure of bio-MOF-14 were prepared sequentially as described above. In this case, there is a pronounced lattice mismatch (bio-MOF-11: $a=b=15.44 \AA, c=22.78 \AA$; bio-MOF14: $a=b=15.85 \AA, c=22.35 \AA$ ) between the two different MOFs, and as a result, the growth of a bio-MOF-14 shell on pristine bio-MOF-11 was not observed. But the mixed core of MOF-11/14 allowed the growth of bio-MOF-14 as a shell structure. The core-shell structure, where the shell structure porosity is less than that of the core structure, demonstrated a $30 \%$ higher uptake of $\mathrm{CO}_{2}$ than did the core crystal.

In recent work, Hecht and coworkers constructed porous core-photoactive shell MOF-on-MOF structures by employing a solvent-assisted linker exchange (SALE) method to modulate the guest uptake by visible light illuminations [61]. In this approach, a UiO-68 MOF was used as a basis structure. Using a 3,3"dihydroxy-2',5'-dimethyl-[1,1':4',1"-terphenyl]-4,4"-dicarboxylic acid ( $\mathrm{L}^{\text {core }}$ ) linker, seed crystals of UiO-68 were synthesized using the solvothermal method. The seed crystals were then used for the SALE process to create a shell UiO-68 structure having a photoswitchable azo group in the linker $\left(\mathrm{L}^{\text {azo }}=2^{\prime}, 5^{\prime}\right.$ dimethyl-2,2"'-bis[4-(2,2',6,6'-tetrafluoroazobenzene)]-[1,1':4',1"terphenyl]-4,4"-dicarboxylic acid) (Figs. 11(b) and 11(c)). In the SALE process, the photoswitchable linker $\mathrm{L}^{\text {azo }}$ is exchanged with the $\mathrm{L}^{\text {core }}$ linkers on the crystal surface, thus creating a shell on the core particles. An uptake experiment of 1-pyrenecarboxylic acid (in ethanol) using the core-shell MOF particles revealed that the cis form of an azobenzene side group showed an $86 \%$ higher uptake than that of the trans form. If the core structure consists of catalytically active sites, this photoswitchable diffusion barrier on the shell of the crystal can also be useful for catalytic applications.

A core-shell architecture, consisting of a magnetic core and a MOF shell, was realized in 2012 [62]. A modified LPE approach was introduced to create such architectures, referred to as magMOFs. In this approach, the magnetic particle (magnetite) is first coated with silica and then used as a core structure. A transmission electron microscopy (TEM) image of such a seed particle is shown in Fig. 12(a). In order to initiate MOF deposition, the silica surface was functionalized with $-\mathrm{COOH}$ end groups, and in a lbl fashion, particles were immersed in metal and linker solutions. To remove the residual metal or linker, particles were washed off with ethanol. The loss of particles when removing the washing solution was avoided by simply applying a magnet. The magMOF approach was (a)
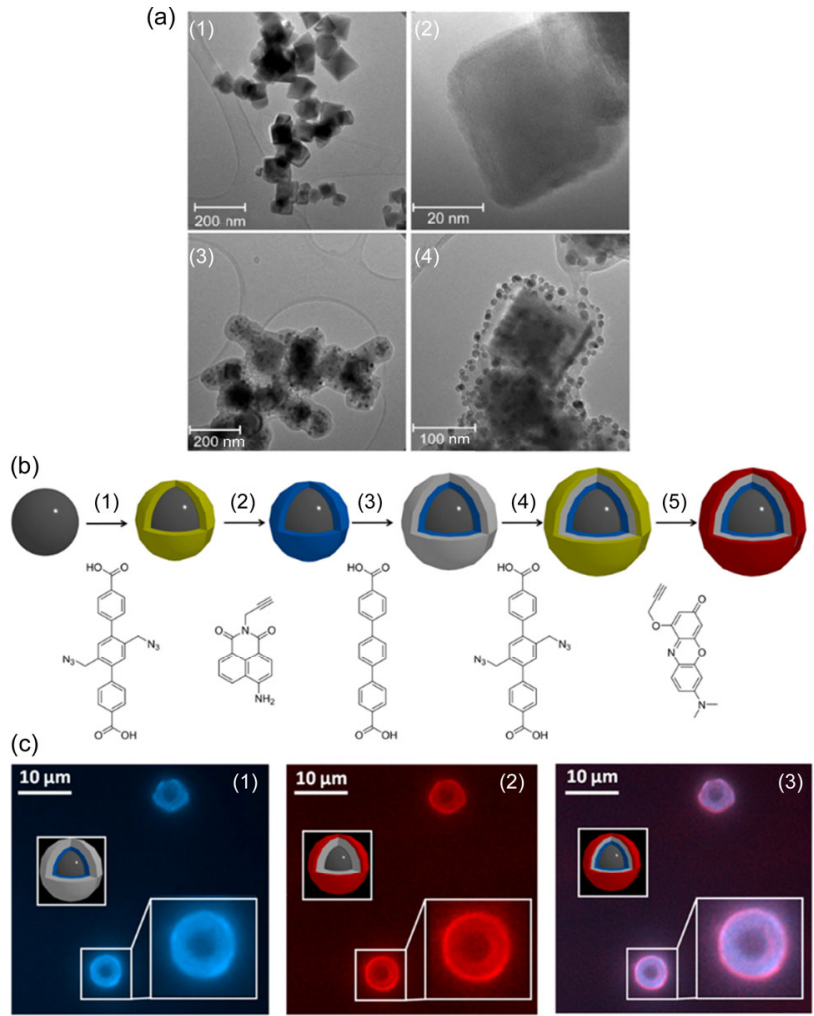

(d)

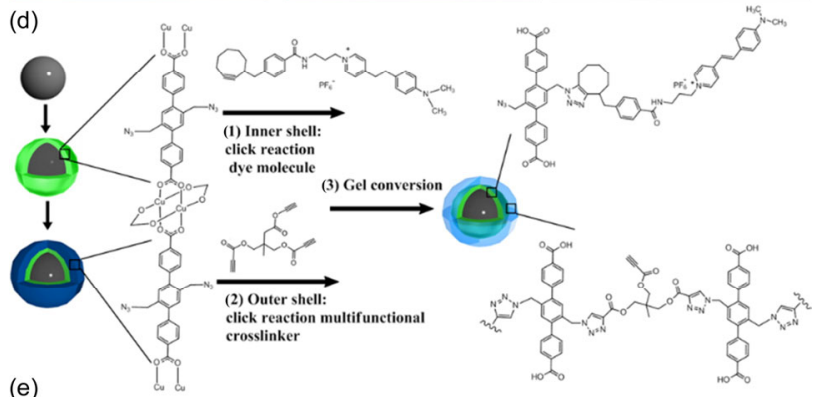

(e)
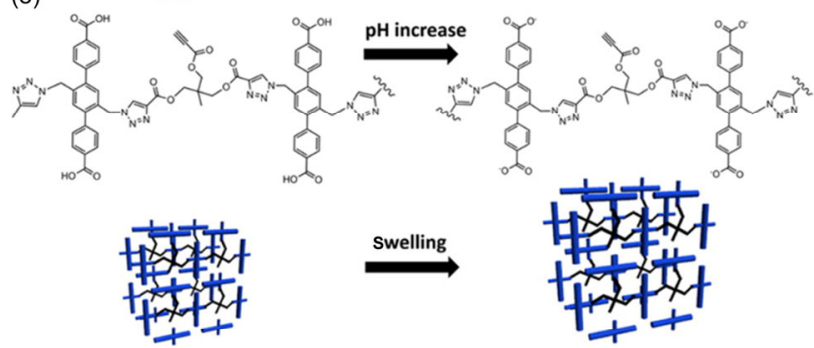

Swelling
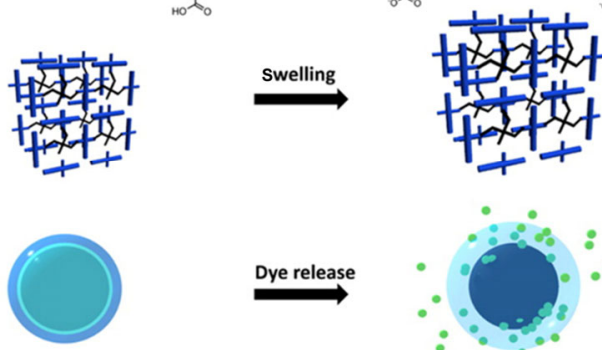

Dye release

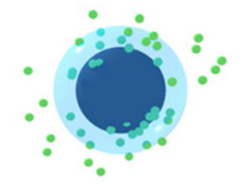

Figure 12 (a) TEM images of the pure MagPrep Silica nanoparticles (top two images) and HKUST-1 grown on COOH terminated MagPrep Silica after 40 cycles of the LPE method (bottom two images) (reproduced with permission from Ref. [62], ( ) Wiley-VCH Verlag GmbH \& Co. KGaA, Weinheim 2013). (b) Different steps of shell-on-shell MOF growth around magnetic core particle: (1) $\mathrm{Cu}(\mathrm{BA}-\mathrm{TPDC}) \mathrm{MOF}$ growth; (2) click reaction of the blue dye; (3) $\mathrm{Cu}$ (TPDC) MOF growth; (4) $\mathrm{Cu}$ (BA-TPDC) MOF growth; (5) click reaction of the red dye; (c) confocal microscope images of the dyefunctionalized multishell magMOF using different filters: (1) blue channel; (2) red channel; (3) overlay of red/blue channel; (d) schematic illustration of the multistep synthesis of dye containing magGEL capsules; (e) illustration of the $\mathrm{pH}$ triggered dye release process from magGel capsules (reproduced with permission from Ref. [63], (c) American Chemical Society 2015).

demonstrated successfully for the case of HKUST-1, ZIF-8, and a pillared-layer MOF $\mathrm{Zn}_{2}(\mathrm{cam})_{2}($ dabco $)(\mathrm{cam}=D$-camphoric 
acid). The phase purity of the magMOF was established by employing XRD measurements, and the surface area of the deposited MOF material was determined by Brunauer-EmmettTeller (BET) measurements. Using these magMOF particles, it is possible to demonstrate chromatographic separation, which is not feasible for SURMOFs grown on planar substrates.

Later, the magMOF approach was also demonstrated for constructing shell-on-shell MOF architectures [63]. In this work, multiple $\mathrm{Cu}$-SURMOF-2 structures having similar unit cell dimensions were coated sequentially on a functionalized magnetic core. The schematic cartoon in Fig. 12(b) illustrates the stepwise construction of the shell-on-shell structure. After growth of the first shell, an emissive dye was covalently functionalized by click reaction. The same was done for second shell structure, as illustrated in Fig. 12(b). The inclusion of the dye molecules in the two different MOF shells was examined by confocal fluorescence microscopy. The corresponding images revealed distinctly separated shell structures (Fig. 12(c)). Following this observation, another shell-on-shell structure was synthesized in which the inner shell was functionalized with a dye again using a click reaction and the outer shell was cross-linked, demonstrated in Fig. 12(d). After cross-linking of the outershell, $\mathrm{Cu}$-metal ions were removed by ethylene diamine tetraacetic acid (EDTA) treatment to create a cross-linked surface gel (SURGEL) outer-shell structure. As the inner shell was not cross-linked, the dye molecules could freely float in the inner layer, as can be seen from the even distribution of dye fluorescence by confocal microscopy. These particles can be used for a controlled release of the dye molecules. In this case, the release is triggered by changing the $\mathrm{pH}$ of the surrounding medium. High $\mathrm{pH}$ values cause a swelling of the SURGEL used as the outer shell. The release of the di-molecules can be easily demonstrated by the color change in the surrounding medium. This gel capsule designed by the magMOF approach can be useful for future drug delivery system design.

A number of other groups have demonstrated the growth of MOF multilayers on particles. Oh et al. started from functionalized silica spheres to grow multiple layers of MOF shell structures by using solvothermal methods in connection with different reactants [64]. In this work, a - $\mathrm{COOH}$ functionalized spherical silica particle was reacted with a metal (Er, Y, or Gd) and linker (isophthalic acid) mixture under solvothermal conditions to homogenously grow a MOF layer as a shell (Fig. 13). This silica@MOF was further used as the core, and a second shell layer of a different metal-MOF was epitaxially grown. All three metal ions yielded isostructural MOF with isophthalic acid.

Oh and coworkers also introduced mixed-metal MOF heterostructures with distinct evidence of isotropic and anisotropic epitaxial growth [65]. In this example, first rod-shaped Fe-MIL-88B MOF particles were grown using the solvothermal method. The Fe-MIL-88B structure is constructed by connecting trimers of $\mathrm{FeO}_{6}$ octahedra with terephthalic acid linker. Subsequently, In and Ga MOF structures were deposited on these seed particles in an epitaxial fashion. Interestingly, In and Ga-MOFs were unable to form in the absence of the Fe-MIL-88B seed crystals (Fig. 14). Microscopic studies and elemental analyses revealed that, in the case of Ga-MOF, a core-shell morphology formed, indicating an isotropic MOFon-MOF growth. In contrast, for In-MOF, only an increase along the length of the rod-shaped crystals was observed. This different growth behavior can be understood from the metal ion size variations which lead to the deposition of the second MOF only on particular surface orientations. The larger size of In(III) compared to Fe(III) led to a different size of trimer formed by $\mathrm{MO}_{6}$ units $(\mathrm{M}=\mathrm{Fe} / \mathrm{In} / \mathrm{Ga})$, and this mismatch

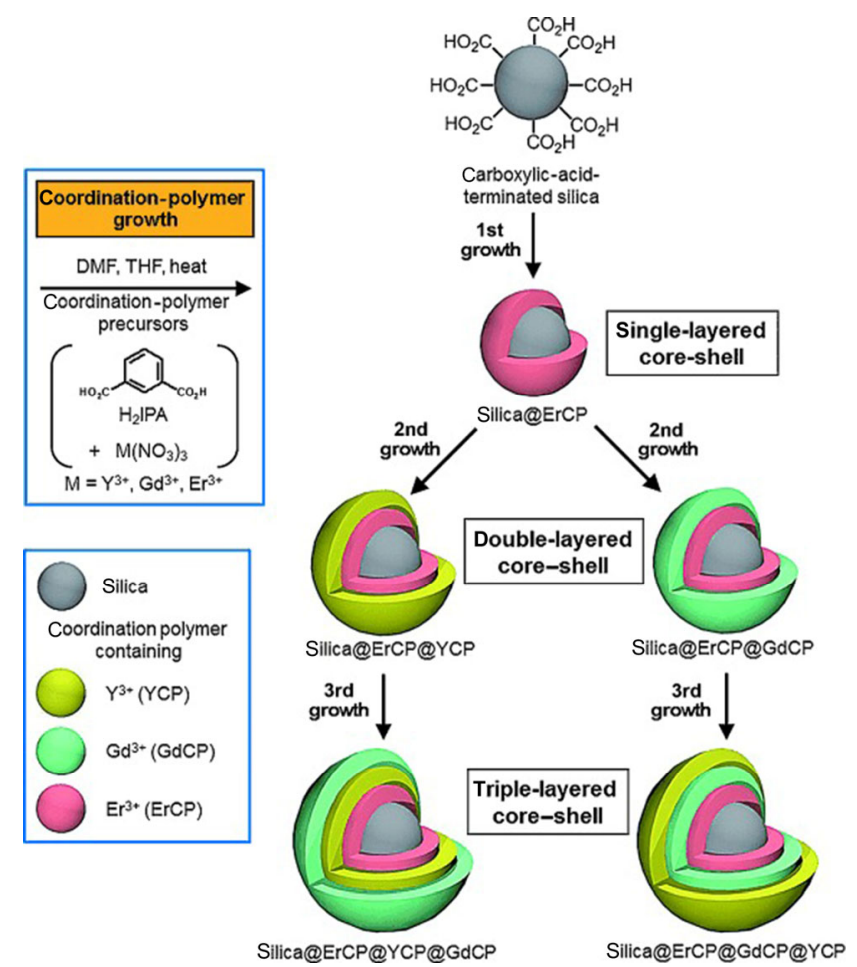

Figure 13 Stepwise growth of multilayered MOF layers in epitaxial fashion as a core-shell structure, supported by functionalized silica spheres (reproduced with permission from Ref. [64], (c) Wiley-VCH Verlag GmbH \& Co. KGaA, Weinheim 2013).

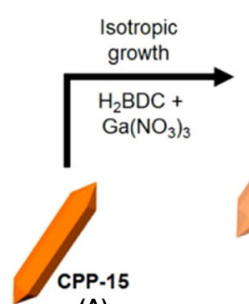

(A)

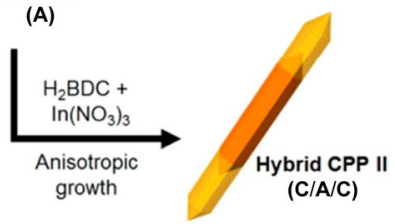

Figure 14 Isotropic and anisotropic growth of MOF-on-MOF: growth of In- and Ga-based MIL-88b MOFs as a shell on a Fe-MIL-88b core crystal and their elemental mapping (right) (reproduced with permission from Ref. [65], (c) American Chemical Society 2015).

restricted the crystal growth in 3D. This anisotropic growth led to a layered heterostructure in the nanoscale MOF crystal.

Subsequently, the same group used In-MIL-68 as the core crystal to demonstrate that secondary, face-selective MOF crystal growth of In-ndc (i.e., a heterocomposition) on such seed crystals is also feasible [65]. On the hexagonal, using rod-shaped In-MIL-68 (linker-terephthalic acid) as seed crystals, isostructural In-MIL-68 with bromoterepthalic acid linker was observed to grow and to yield a shell structure, strongly suggesting isotropic epitaxial growth. However, changing the linker of the secondary MOF to ndc (identical in length to terephthalic acid) yielded a quite different morphology. Instead of a uniform coating, the formation of a semitubular crystal morphology was observed. This was explained by the difference in crystal structure formed by terephthalic acid and ndc with In(III). The ndc linker with $\operatorname{In}($ III) in a solvothermal condition did not produce a MIL-68 analogous structure, and hence, In-ndc did not grow isotropically on the 
In-MIL-68 crystal seed. However, the rectangular facet of In-MIL-68 had a similar lattice dimension as the In-ndc structure, and hence, the shell structure grows selectively.

More recently, Zhou et al. reported that hybrid core-shell MOF particles can be synthesized even in the presence of strong lattice mismatches and for a different symmetries by precisely controlling the nucleation kinetics [66]. A tetracarboxylate tcpp linker (tcpp = tetrakis (4-carboxyphenyl porphyrin)) nucleates faster than a dicarboxylate bpdc linker due to the faster coordination kinetics with $\mathrm{Zr}^{4+}$. Mixing these two linkers and a metal precursor in one-pot synthesis, tcpp-MOF nucleates faster and then heterogeneous MOF-on-MOF growth proceeds, i.e., the growth of the shell structure of bpdc-MOF (Fig. 15(a)). Separate crystal growth of two individual MOFs (i.e., homogeneous growth) is kinetically very slow, as the seed crystal of tcpp-MOF acts as a template. Using this method, a PCN222@Zr-bpdc hybrid MOF was fabricated with mesoporous core and microporous shell structure. By constructing a core-shell

(a)
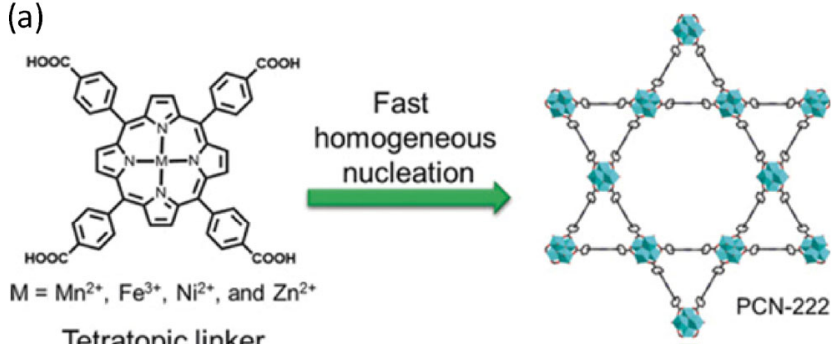

Tetratopic linker
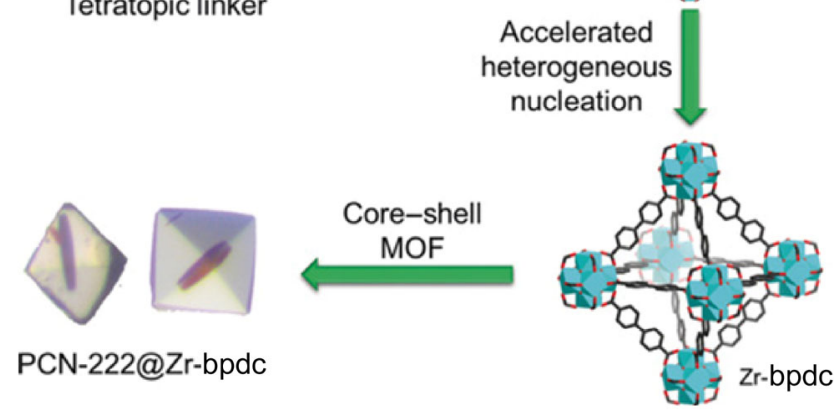

PCN-222@Zr-bpdc

(b)

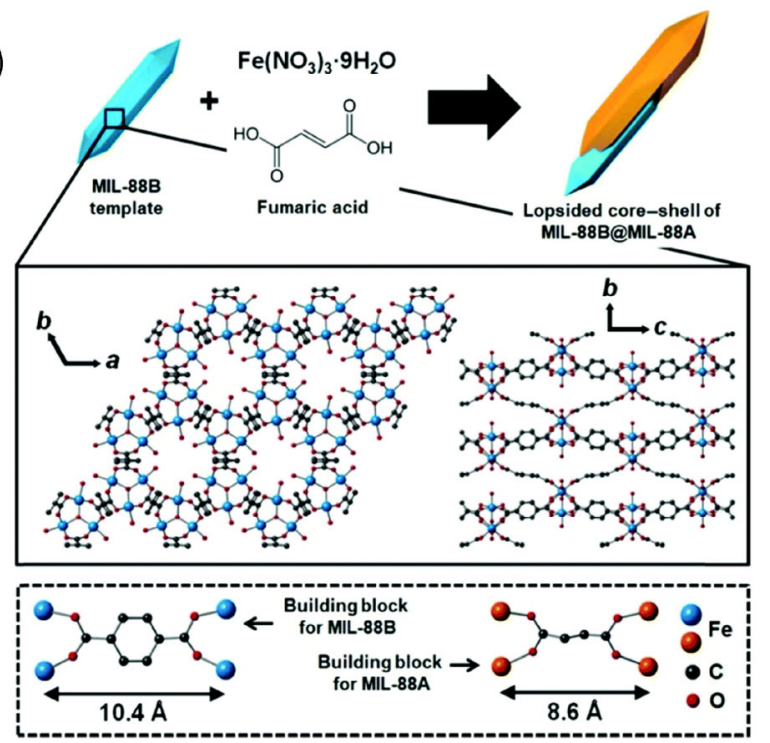

Figure 15 (a) Illustration of kinetically controlled core-shell MOF-on-MOF growth with mismatched lattice dimensions (reproduced with permission from Ref. [66], ๑ Wiley-VCH Verlag GmbH \& Co. KGaA, Weinheim 2018). (b) Lopsided core-shell MOF-on-MOF heterostructure grown in a stepwise method (reproduced with permission from Ref. [32], @ The Royal Society of Chemistry 2019). structure PCN222 (Fe)@Zr-bpdc, a size-selective catalysis for epoxidation of alkenes was realized. Heterogeneous epitaxial MOF growth was also demonstrated by Oh et al. for Fe-MIL88b@Fe-MIL-88a structure, which exhibited an unusual core-shell structure [67]. Hexagonal rods of Fe-MIL-88b MOF crystals (1,4-benzenedicarboxylate linker) were used as a core, and Fe-MIL-88a (fumaric acid linker) structure was grown as a shell. A significant difference in linker length resulted in a lopsided epitaxial growth, as shown in Fig. 15(b).

Using stepwise methods employing kinetic control, it has been possible to create hybrid structures with precise core-shell or block-shaped morphology also with solvothermal synthesis protocols. In most of these cases, the studies focused on the identification of the growth mechanism. In only a few cases was the functionality of such heterostructures investigated. In this context, Kitagawa et al. elegantly demonstrated the advantages of a sequentially functionalized MOF structure by the MOF-onMOF approach [68]. A combination of multiple functionality was derived by using isoreticular epitaxial growth in a hybrid MOF system, as shown in Fig. 16. A MOF-on-MOF architecture was obtained by sequential solvothermal synthesis steps, as illustrated before. Using a porous container MOF as a core structure and sieving MOF as a shell structure, extraction of cetane from isocetane at a very low concentration of cetane $(<1 \%)$ was demonstrated. Indeed, such superior function in these hybrid structures will direct many other applications in the direction of mass transport, sensing, etc.

In the context of screening of the possibilities of MOF heterostructure growth, recently a combinatorial computation/ experimental workflow was presented by Kim et al. [69]. Considering the vast amount of different MOF topologies, it is indeed an attractive strategy to isolate relevant heterostructures. On the basis of two important parameters, i) lattice parameters are nearly identical or multiples of one another, and ii) interfaces

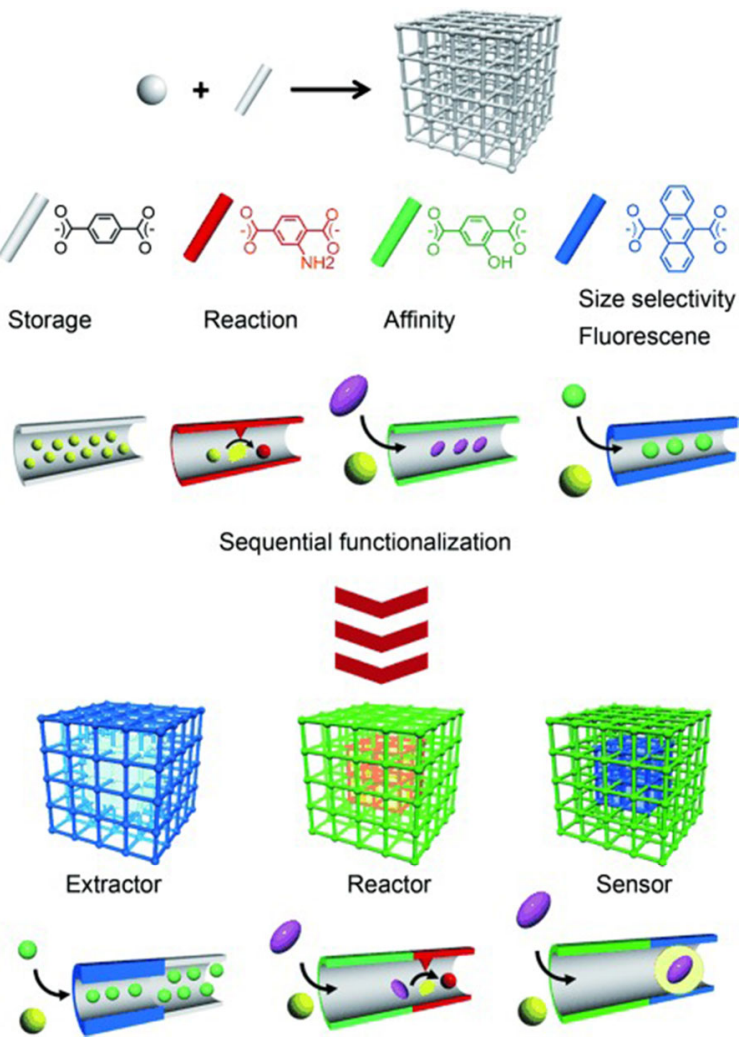

Figure 16 A schematic illustration of sequential functionalization of a hybrid hierarchical MOF structure using epitaxial growth of isoreticular MOFs (reproduced with permission from Ref. [68], ( ) Wiley-VCH Verlag GmbH \& Co. KGaA, Weinheim 2011). 
possess well-matched chemical connection points, an algorithm has been developed to screen hundreds of MOF pairs. Among these, 6 pairs of MOF heterostructures HKUST-1@MOF-5, HKUST-1@IRMOF-18, UiO-67@HKUST-1, PCN-68@MOF-5, UiO-66@MIL-88B(Fe) and UiO-67@MIL-88C(Fe) were found suitable and could be successfully synthesized as a single crystalline MOF@MOF material. This demonstration of rapid screening is an important step forward for the realization of functional heterostructures, and can be employed for more sophisticated lbl approach.

\section{Outlook}

While conceiving advanced heterostructures for multifunctional materials systems using multicomponent MOFs is very appealing, realizing structurally well-defined, hierarchical assemblies with low defect densities using conventional, solvothermal MOF synthesis methods is a major challenge. The realization of such architectures is largely simplified by the lbl process, as illustrated in this review. The SURMOF approach has been successful in addressing the precise control of thickness and homogeneity of the MOF layers, overcoming challenges in the growth process of mismatched lattice structures and, more importantly, producing high-quality organic-organic interfaces with low densities of defects. Optoelectronic properties such as photon upconversion, energy transfer, and exciton diffusion anisotropy have been recently explored using such heterolayer SURMOF structures, which indicated the great promise of these emerging hierarchically structured materials. Meanwhile, core-shell or block-layer type MOF-on-MOF heterostructures have also been designed by the conventional MOF growth approach. For the step-by-step growth of core and shell or kinetically controlled nucleation, both strategies are attractive and can address challenges concerning efficient mass transport, selective encapsulation, size-selective catalysis, and controlled release. The major difficulties with regard to characterizations and control over the layer size/thickness in such core-shell structures can be addressed by the lbl method. In addition to MOF-on-MOF hybrids, MOF-on-oxide/polymer type architectures are also attractive from the viewpoint of device-based applications. A rather unique case is the magMOF approach, where particle separation is straightforward and even application in chromatographic separation is feasible. Already, the existing literature shows the great promise of the lbl methodology in this regard, and in the future, advanced MOF-hybrid hierarchical design will rely heavily on a similar methodology to address technological challenges.

With regard to the screening of the possible functional materials, a combinatorial approach is of great value. We foresee that the future development of MOF heterostructures will make more use of simulations to explore the potential of the huge number of MOFs available. Among the aspects to be considered in such screening efforts are the possibility of epitaxial growth and the control of defect density at the hetero-interface. Also the design of multi-heterolayers with more than two different components to enter the field of photonic crystals will become easier when supported by theoretical work. Applications of MOFs in the field of energy and electronics will require facile integration of MOF heterostructures in devices, which also calls for the optimization of interfaces between MOFs to other materials, which represents a major challenge. We also expect that the combination of MOF-on-MOF growth with lithographical methods, which is straightforward in combination with the SURMOF approach, will become an exciting topic in the next years.

\section{Acknowledgements}

The authors acknowledge support from Deutsche Forschungsgemeinschaft (DFG, German Research Foundation) under Germany's Excellence Strategy-2082/1-390761711 and SPP 1928 "COORNETS." We thank Xiaojing Liu for comments on the manuscript.

Funding note: Open access funding provided by Projekt DEAL

Open Access This article is licensed under a Creative Commons Attribution 4.0 International License, which permits use, sharing, adaptation, distribution and reproduction in any medium or format, as long as you give appropriate credit to the original author(s) and the source, provide a link to the Creative Commons licence, and indicate if changes were made.

The images or other third party material in this article are included in the article's Creative Commons licence, unless indicated otherwise in a credit line to the material. If material is not included in the article's Creative Commons licence and your intended use is not permitted by statutory regulation or exceeds the permitted use, you will need to obtain permission directly from the copyright holder.

To view a copy of this licence, visit http://creativecommons.org/licenses/by/4.0/.

\section{References}

[1] Yaghi, O. M. Reticular chemistry in all dimensions. ACS Cent. Sci. 2019, 5, 1295-1300.

[2] Ockwig, N. W.; Delgado-Friedrichs, O.; O'Keeffe, M.; Yaghi, O. M. Reticular chemistry: Occurrence and taxonomy of nets and grammar for the design of frameworks. Acc. Chem. Res. 2005, 38, 176-182.

[3] Kitagawa, S.; Kitaura, R.; Noro, S. I. Functional porous coordination polymers. Angew. Chem., Int. Ed. 2004, 43, 2334-2375.

[4] Haldar, R.; Maji, T. K. Metal-organic frameworks (MOFs) based on mixed linker systems: Structural diversities towards functional materials. CrystEngComm 2013, 15, 9276-9295.

[5] Haldar, R.; Sikdar, N.; Maji, T. K. Interpenetration in coordination polymers: Structural diversities toward porous functional materials. Mater. Today 2015, 18, 97-116.

[6] Farha, O. K.; Eryazici, I.; Jeong, N. C.; Hauser, B. G.; Wilmer, C. E.; Sarjeant, A. A.; Snurr, R. Q.; Nguyen, S. T.; Yazaydın, A. Ö.; Hupp, J. T. Metal-organic framework materials with ultrahigh surface areas: Is the sky the limit? J. Am. Chem. Soc. 2012, 134, 15016-15021.

[7] Suh, M. P.; Park, H. J.; Prasad, T. K.; Lim, D. W. Hydrogen storage in metal-organic frameworks. Chem. Rev. 2012, 112, 782-835.

[8] Li, B.; Wen, H. M.; Zhou, W.; Chen, B. L. Porous metal-organic frameworks for gas storage and separation: What, how, and why? J. Phys. Chem. Lett. 2014, 5, 3468-3479.

[9] Zhao, X.; Wang, Y. X.; Li, D. S.; Bu, X. H.; Feng, P. Y. Metalorganic frameworks for separation. Adv. Mater. 2018, 30, 1705189.

[10] Hu, Z. G.; Wang, Y. X.; Shah, B. B.; Zhao, D. $\mathrm{CO}_{2}$ capture in metalorganic framework adsorbents: An engineering perspective. $A d v$. Sustain. Syst. 2019, 3, 1800080.

[11] Huang, Y. B.; Liang, J.; Wang, X. S.; Cao, R. Multifunctional metalorganic framework catalysts: Synergistic catalysis and tandem reactions. Chem. Soc. Rev. 2017, 46, 126-157.

[12] Zhu, L.; Liu, X. Q.; Jiang, H. L.; Sun, L. B. Metal-organic frameworks for heterogeneous basic catalysis. Chem. Rev. 2017, 117, 81298176.

[13] Stavila, V.; Talin, A. A.; Allendorf, M. D. MOF-based electronic and opto-electronic devices. Chem. Soc. Rev. 2014, 43, 5994-6010.

[14] Haldar, R.; Heinke, L.; Wöll, C. Advanced photoresponsive materials using the metal-organic framework approach. Adv. Mater. 2020, 32, 1905227.

[15] Dolgopolova, E. A.; Rice, A. M.; Martin, C. R.; Shustova, N. B. Photochemistry and photophysics of MOFs: Steps towards MOF-based 
sensing enhancements. Chem. Soc. Rev. 2018, 47, 4710-4728.

[16] Lustig, W. P.; Mukherjee, S.; Rudd, N. D.; Desai, A. V.; Li, J.; Ghosh, S. K. Metal-organic frameworks: Functional luminescent and photonic materials for sensing applications. Chem. Soc. Rev. 2017, $46,3242-3285$.

[17] Begum, S.; Hassan, Z.; Bräse, S.; Wöll, C.; Tsotsalas, M. Metalorganic framework-templated biomaterials: Recent progress in synthesis, functionalization, and applications. Acc. Chem. Res. 2019, $52,1598-1610$.

[18] Lismont, M.; Dreesen, L.; Wuttke, S. Metal-organic framework nanoparticles in photodynamic therapy: Current status and perspectives. Adv. Funct. Mater. 2017, 27, 1606314.

[19] Eddaoudi, M.; Kim, J.; Rosi, N.; Vodak, D.; Wachter, J.; O'Keeffe, M.; Yaghi, O. M. Systematic design of pore size and functionality in isoreticular MOFs and their application in methane storage. Science 2002, 295, 469-472.

[20] Deng, H. X.; Doonan, C. J.; Furukawa, H.; Ferreira, R. B.; Towne, J.; Knobler, C. B.; Wang, B.; Yaghi, O. M. Multiple functional groups of varying ratios in metal-organic frameworks. Science 2010, 327, 846-850.

[21] Deng, H. X.; Grunder, S.; Cordova, K. E.; Valente, C.; Furukawa, H.; Hmadeh, M.; Gándara, F.; Whalley, A. C.; Liu, Z.; Asahina, S. et al. Large-pore apertures in a series of metal-organic frameworks. Science 2012, 336, 1018-1023.

[22] Wang, Z. B.; Liu, J. X.; Grosjean, S.; Wagner, D.; Guo, W.; Gu, Z. G.; Heinke, L.; Gliemann, H.; Bräse, S.; Wöll, C. Monolithic, crystalline MOF coating: An excellent patterning and photoresist material. ChemNanoMat 2015, 1, 338-345.

[23] Gliemann, H.; Wöll, C. Epitaxially grown metal-organic frameworks. Mater. Today 2012, 15, 110-116.

[24] Shekhah, O.; Wang, H.; Kowarik, S.; Schreiber, F.; Paulus, M.; Tolan, M.; Sternemann, C.; Evers, F.; Zacher, D.; Fischer, R. A. et al. Step-by-step route for the synthesis of metal-organic frameworks. J. Am. Chem. Soc. 2007, 129, 15118-15119.

[25] Liu, J. X.; Wöll, C. Surface-supported metal-organic framework thin films: Fabrication methods, applications, and challenges. Chem. Soc. Rev. 2017, 46, 5730-5770.

[26] Shekhah, O.; Liu, J.; Fischer, R. A.; Wöll, C. MOF thin films: Existing and future applications. Chem. Soc. Rev. 2011, 40, 1081-1106.

[27] Kong, X. Q.; Deng, H. X.; Yan, F. Y.; Kim, J.; Swisher, J. A.; Smit, B.; Yaghi, O. M.; Reimer, J. A. Mapping of functional groups in metalorganic frameworks. Science 2013, 341, 882-885.

[28] Furukawa, S.; Hirai, K.; Nakagawa, K.; Takashima, Y.; Matsuda, R.; Tsuruoka, T.; Kondo, M.; Haruki, R.; Tanaka, D.; Sakamoto, H. et al. Heterogeneously hybridized porous coordination polymer crystals: Fabrication of heterometallic core-shell single crystals with an in-plane rotational epitaxial relationship. Angew. Chem., Int. Ed. 2009, 48, $1766-1770$

[29] Liu, J. X.; Lukose, B.; Shekhah, O.; Arslan, H. K.; Weidler, P.; Gliemann, H.; Bräse, S.; Grosjean, S.; Godt, A.; Feng, X. L. et al. A novel series of isoreticular metal organic frameworks: Realizing metastable structures by liquid phase epitaxy. Sci. Rep. 2012, 2, 921.

[30] Valadez Sánchez, E. P.; Gliemann, H.; Haas-Santo, K.; Wöll, C.; Dittmeyer, R. ZIF-8 SURMOF membranes synthesized by Au-assisted liquid phase epitaxy for application in gas separation. Chem. Ingen. Tech. 2016, 88, 1798-1805.

[31] Hashem, T.; Valadez Sánchez, E. P.; Weidler, P. G.; Gliemann, H.; Alkordi, M. H.; Wöll, C. Front cover: Liquid-phase quasi-epitaxial growth of highly stable, monolithic UiO-66- $\mathrm{NH}_{2}$ MOF thin films on solid substrates (ChemistryOpen 5/2020). ChemistryOpen 2020, 9, 521.

[32] Shekhah, O.; Hirai, K.; Wang, H.; Uehara, H.; Kondo, M.; Diring, S.; Zacher, D.; Fischer, R. A.; Sakata, O.; Kitagawa, S. et al. MOF-onMOF heteroepitaxy: Perfectly oriented $\left[\mathrm{Zn}_{2}(\mathrm{ndc})_{2}(\mathrm{dabco})\right]_{n}$ grown on $\left[\mathrm{Cu}_{2}(\mathrm{ndc})_{2}(\mathrm{dabco})\right]_{n}$ thin films. Dalton Trans. 2011, 40, 49544958.

[33] Chun, H.; Dybtsev, D. N.; Kim, H.; Kim, K. Synthesis, X-ray crystal structures, and gas sorption properties of pillared square grid nets based on paddle-wheel motifs: Implications for hydrogen storage in porous materials. Chem.-Eur. J. 2005, 11, 3521-3529.

[34] Seki, K.; Mori, W. Syntheses and characterization of microporous coordination polymers with open frameworks. J. Phys. Chem. B 2002,
$106,1380-1385$.

[35] Tang, S. J.; Lee, C. Y.; Huang, C. C.; Chang, T. R.; Cheng, C. M.; Tsuei, K. D.; Jeng, H. T.; Yeh, V.; Chiang, T. C. Electronic versus lattice match for metal-semiconductor epitaxial growth: $\mathrm{Pb}$ on Ge(111). Phys. Rev. Lett. 2011, 107, 066802.

[36] Li, H. L.; Eddaoudi, M.; Groy, T. L.; Yaghi, O. M. Establishing microporosity in open metal-organic frameworks: Gas sorption isotherms for $\mathrm{Zn}(\mathrm{BDC})(\mathrm{BDC}=1,4$-benzenedicarboxylate). J. Am. Chem. Soc. 1998, 120, 8571-8572.

[37] Haldar, R.; Batra, K.; Marschner, S. M.; Kuc, A. B.; Zahn, S.; Fischer, R. A.; Bräse, S.; Heine, T.; Wöll, C. Bridging the green gap: Metal-organic framework heteromultilayers assembled from porphyrinic linkers identified by using computational screening.. Chem.-Eur. J. 2019, 25, 7847-7851.

[38] Haldar, R.; Mazel, A.; Joseph, R.; Adams, M.; Howard, I. A.; Richards, B. S.; Tsotsalas, M.; Redel, E.; Diring, S.; Odobel, F. et al. Excitonically coupled states in crystalline coordination networks. Chem.-Eur. J. 2017, 23, 14316-14322.

[39] Liu, J. X.; Zhou, W. C.; Liu, J. X.; Howard, I.; Kilibarda, G.; Schlabach, S.; Coupry, D.; Addicoat, M.; Yoneda, S.; Tsutsui, Y. et al. Photoinduced charge-carrier generation in epitaxial MOF thin films: High efficiency as a result of an indirect electronic band gap? Angew. Chem., Int. Ed. $\mathbf{2 0 1 5}, 54,7441-7445$.

[40] Liu, J. X.; Zhou, W. C.; Liu, J. X.; Fujimori, Y.; Higashino, T.; Imahori, H.; Jiang, X.; Zhao, J. J.; Sakurai, T.; Hattori, Y. et al. A new class of epitaxial porphyrin metal-organic framework thin films with extremely high photocarrier generation efficiency: Promising materials for all-solid-state solar cells. J. Mater. Chem. A 2016, 4, 12739-12747.

[41] Haldar, R.; Diring, S.; Samanta, P. K.; Muth, M.; Clancy, W.; Mazel, A.; Schlabach, S.; Kirschhöfer, F.; Brenner-Weiß, G.; Pati, S. K. et al. Enhancing selectivity and kinetics in oxidative photocyclization by supramolecular control. Angew. Chem., Int. Ed. 2018, 57, 13662-13665.

[42] Wang, Z. B.; Liu, J. X.; Lukose, B.; Gu, Z. G.; Weidler, P. G.; Gliemann, H.; Heine, T.; Wöll, C. Nanoporous designer solids with huge lattice constant gradients: Multiheteroepitaxy of metal-organic frameworks. Nano Lett. 2014, 14, 1526-1529.

[43] Wang, Z.; Wannapaiboon, S.; Rodewald, K.; Tu, M.; Rieger, B.; Fischer, R. A. Directing the hetero-growth of lattice-mismatched surfacemounted metal-organic frameworks by functionalizing the interface. J. Mater. Chem. A 2018, 6, 21295-21303.

[44] Tu, M.; Fischer, R. A. Heteroepitaxial growth of surface mounted metal-organic framework thin films with hybrid adsorption functionality. J. Mater. Chem. A 2014, 2, 2018-2022.

[45] Heinke, L.; Cakici, M.; Dommaschk, M.; Grosjean, S.; Herges, R.; Bräse, S.; Wöll, C. Photoswitching in two-component surface-mounted metalorganic frameworks: Optically triggered release from a molecular container. ACS Nano 2014, 8, 1463-1467.

[46] Meilikhov, M.; Furukawa, S.; Hirai, K.; Fischer, R. A.; Kitagawa, S. Binary janus porous coordination polymer coatings for sensor devices with tunable analyte affinity. Angew. Chem., Int. Ed. 2013, 52, 341-345.

[47] Ikigaki, K.; Okada, K.; Tokudome, Y.; Toyao, T.; Falcaro, P.; Doonan, C. J.; Takahashi, M. MOF-on-MOF: Oriented growth of multiple layered thin films of metal-organic frameworks. Angew. Chem., Int. Ed. 2019, 58, 6886-6890.

[48] Falcaro, P.; Okada, K.; Hara, T.; Ikigaki, K.; Tokudome, Y.; Thornton, A. W.; Hill, A. J.; Williams, T.; Doonan, C.; Takahashi, M. Centimetre-scale micropore alignment in oriented polycrystalline metal-organic framework films via heteroepitaxial growth. Nat. Mater. 2017, 16, 342-348.

[49] Raišys, S.; Kazlauskas, K.; Juršènas, S.; Simon, Y. C. The role of triplet exciton diffusion in light-upconverting polymer glasses. ACS Appl. Mater. Interfaces 2016, 8, 15732-15740.

[50] Oldenburg, M.; Turshatov, A.; Busko, D.; Wollgarten, S.; Adams, M.; Baroni, N.; Welle, A.; Redel, E.; Wöll, C.; Richards, B. S. et al. Photon upconversion at crystalline organic-organic heterojunctions. Adv. Mater. 2016, 28, 8477-8482.

[51] Haldar, R.; Jakoby, M.; Mazel, A.; Zhang, Q.; Welle, A.; Mohamed, T.; Krolla, P.; Wenzel, W.; Diring, S.; Odobel, F. et al. Anisotropic energy transfer in crystalline chromophore assemblies. Nat. Commun. 2018, 9, 4332 . 
[52] Haldar, R.; Batra, K.; Marschner, S. M.; Kuc, A. B.; Zahn, S.; Fischer, R. A.; Bräse, S.; Heine, T.; Wöll, C. Bridging the green gap: Metal-organic framework heteromultilayers assembled from porphyrinic linkers identified by using computational screening. Chem.-Eur. J. 2019, 25, 7847-7851.

[53] Park, H. J.; So, M. C.; Gosztola, D.; Wiederrecht, G. P.; Emery, J. D.; Martinson, A. B. F.; Er, S.; Wilmer, C. E.; Vermeulen, N. A.; AspuruGuzik, A. et al. Layer-by-layer assembled films of perylene diimideand Squaraine-containing metal-organic framework-like materials: Solar energy capture and directional energy transfer. ACS Appl. Mater. Interfaces 2016, 8, 24983-24988.

[54] Chen, D. H.; Haldar, R.; Neumeier, B. L.; Fu, Z. H.; Feldmann, C.; Wöll, C.; Redel, E. Tunable emission in heteroepitaxial Ln-SURMOFs. Adv. Funct. Mater. 2019, 29, 1903086.

[55] Rocha, J.; Carlos, L. D.; Paz, F. A. A.; Ananias, D. Luminescent multifunctional lanthanides-based metal-organic frameworks. Chem. Soc. Rev. 2011, 40, 926-940.

[56] Mohapatra, S.; Adhikari, S.; Riju, H.; Maji, T. K. Terbium(III), europium(III), and mixed terbium(III)-europium(III) mucicate frameworks: Hydrophilicity and stoichiometry-dependent color tunability. Inorg. Chem. 2012, 51, 4891-4893.

[57] Liu, J. X.; Redel, E.; Walheim, S.; Wang, Z. B.; Oberst, V.; Liu, J. X.; Heissler, S.; Welle, A.; Moosmann, M.; Scherer, T. et al. Monolithic high performance surface anchored metal-organic framework Bragg reflector for optical sensing. Chem Mater. 2015, 27, 1991-1996.

[58] Zhang, Z. J.; Müller, K.; Heidrich, S.; Koenig, M.; Hashem, T.; Schlöder, T.; Bléger, D.; Wenzel, W.; Heinke, L. Light-switchable one-dimensional photonic crystals based on MOFs with photomodulatable refractive index. J. Phys. Chem. Lett. 2019, 10, 6626-6633.

[59] Furukawa, S.; Hirai, K.; Takashima, Y.; Nakagawa, K.; Kondo, M.; Tsuruoka, T.; Sakata, O.; Kitagawa, S. A block PCP crystal: Anisotropic hybridization of porous coordination polymers by face-selective epitaxial growth. Chem. Commun. 2009, 5097-5099.
[60] Li, T.; Sullivan, J. E.; Rosi, N. L. Design and preparation of a core-shell metal-organic framework for selective $\mathrm{CO}_{2}$ capture. $J$. Am. Chem. Soc. 2013, 135, 9984-9987.

[61] Mutruc, D.; Goulet-Hanssens, A.; Fairman, S.; Wahl, S.; Zimathies, A.; Knie, C.; Hecht, S. Modulating guest uptake in core-shell MOFs with visible light. Angew. Chem., Int. Ed. 2019, 58, 12862-12867.

[62] Silvestre, M. E.; Franzreb, M.; Weidler, P. G.; Shekhah, O.; Wöll, C. Magnetic cores with porous coatings: Growth of metal-organic frameworks on particles using liquid phase epitaxy. Adv. Funct. Mater. 2013, 23, 1210-1213.

[63] Schmitt, S.; Silvestre, M.; Tsotsalas, M.; Winkler, A. L.; Shahnas, A.; Grosjean, S.; Laye, F.; Gliemann, H.; Lahann, J.; Bräse, S. et al. Hierarchically functionalized magnetic core/multishell particles and their postsynthetic conversion to polymer capsules. ACS Nano $\mathbf{2 0 1 5}$, 9, 4219-4226.

[64] Son, J.; Lee, H. J.; Oh, M. Systematic formation of multilayered core-shell microspheres through the multistep growth of coordination polymers. Chem.-Eur. J. 2013, 19, 6546-6550.

[65] Lee, H. J.; Cho, Y. J.; Cho, W.; Oh, M. Controlled isotropic or anisotropic nanoscale growth of coordination polymers: Formation of hybrid coordination polymer particles. ACS Nano 2013, 7, 491-499.

[66] Yang, X. Y.; Yuan, S.; Zou, L. F.; Drake, H.; Zhang, Y. M.; Qin, J. S.; Alsalme, A.; Zhou, H. C. One-step synthesis of hybrid core-shell metalorganic frameworks. Angew. Chem., Int. Ed. 2018, 57, 3927-3932.

[67] Kim, D.; Lee, G.; Oh, S.; Oh, M. Unbalanced MOF-on-MOF growth for the production of a lopsided core-shell of MIL-88B@MIL-88A with mismatched cell parameters. Chem. Commun. 2019, 55, 43-46.

[68] Hirai, K.; Furukawa, S.; Kondo, M.; Uehara, H.; Sakata, O.; Kitagawa, S. Sequential functionalization of porous coordination polymer crystals. Angew. Chem., Int. Ed. 2011, 50, 8057-8061.

[69] Kwon, O.; Kim, J. Y.; Park, S.; Lee, J. H.; Ha, J.; Park, H.; Moon, H. R.; Kim, J. Computer-aided discovery of connected metal-organic frameworks. Nat. Commun. 2019, 10, 3620. 